\title{
First Insight on Small Molecules as Cardiac Calsequestrin Stabilizers
}

Harapriya Chakravarty ${ }^{a}$, Chandralata Bal ${ }^{a}$, Monika Yadav ${ }^{a}$, Nivedita Jena ${ }^{b}$, Naresh C. Balc,*, and Ashoke Sharon $^{a^{*}}$

aDepartment of Chemistry, BIT Mesra, Ranchi 835215, INDIA

${ }^{b}$ KIIT Technology Business Incubator, KIIT University, Bhubaneswar 75102,1 INDIA

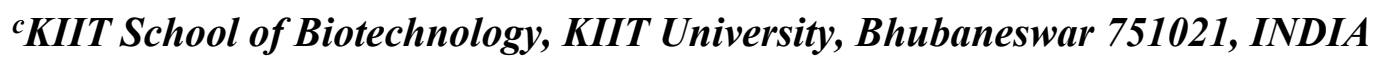

*Corresponding Authors

Naresh Chandra Bal

Phone: +91-9556716478

Email: nareshbal.osu@gmail.com

Mailing address: KIIT School of Biotechnology, KIIT University, Bhubaneswar 751021, India

Ashoke Sharon

Phone: $+91-9470959291$

Email: asharon@bitmesra.ac.in

Mailing address: Department of Chemistry, BIT Mesra, Ranchi 835215, India 


\section{SUPPORTING INFORMATION}

\section{CHEMISTRY}

General: All the reactions were carried out in oven-dried glassware. The chemicals and solvents were purchased from Sigma-Aldrich, Spectrochem, or Acros. Reagents and solvents were used without any purification except THF which was dried by refluxing over sodium followed by distillation. Reactions were monitored by TLC,

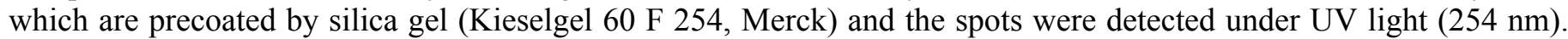
Compounds were purified by column chromatography using silica gel (particle size $100-200$ mesh). ${ }^{1} \mathrm{H}$ and ${ }^{13} \mathrm{C}$ NMR spectra were characterized in $\mathrm{CDCl}_{3}$ or DMSO- $\mathrm{d}_{6}$ solution by using (Wormhole-vnmrs/ Bruker/ Jeol-Delta/Varian) $400 \mathrm{MHz} / 300 \mathrm{MHz}$ spectrophotometers. Chemical shifts are reported as ppm $(\delta)$ relative to TMS $(\delta 0.0)$ as internal standard. ${ }^{1} \mathrm{H}$ NMR data is recorded as follows: chemical shift [multiplicity, coupling constant(s) $J(\mathrm{~Hz})$, relative integral] where multiplicity is defined as: $\mathrm{s}$ (singlet), $\mathrm{d}$ (doublet), t (triplet), q (quartet), m (multiplet), bs (broad singlet). Mass spectra were recorded on 6430 Triple quardrupole mass spectrometer for ESI and are given in mass units Mass-to-charge, (m/z). FTIR spectra were recorded on a Perkin-Elmer 1600 series FT-IR spectrophotometer. UV spectra were recorded on a Perkin Elmer, USA, Lambda 25 UV-Visible spectrophotometer.

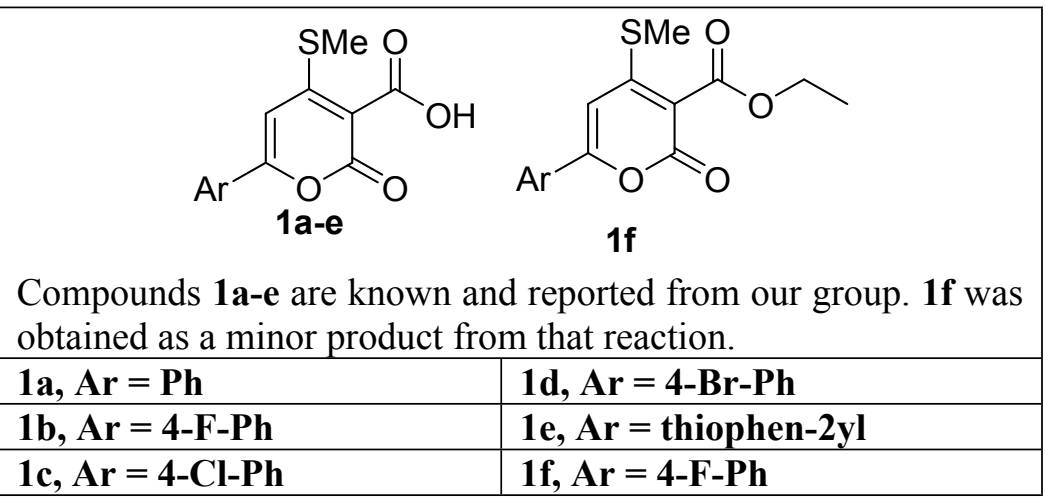<smiles>CCOC(=O)c1c(SC)cc(-c2ccc(F)cc2)oc1=O</smiles>

Scheme S1: Preparation of 6-(4-fluorophenyl)-4-(methylthio)-2-oxo-2H-pyran-3-carboxylic acid (1b) and ethyl 6-(4fluorophenyl)-4-(methylthio)-2-oxo-2H-pyran-3-carboxylate (1f)

i) $\mathrm{NaH}$, diethylmalonate, dioxane, $0-110^{\circ} \mathrm{C}, 4 \mathrm{~h}$

General Procedure: To the stirred suspension of $\mathrm{NaH}(0.066 \mathrm{~mol})$ in dioxane, diethyl malonate $(0.029 \mathrm{~mol})$ was added drop wise. The reaction mixture turned into a thick gel which was stirred for $30 \mathrm{~min}$ at $5^{\circ} \mathrm{C}$ and $1-(4-$ fluorophenyl)-3,3-bis(methylthio)prop-2-en-1-one $(0.0267 \mathrm{~mol})$ was added. It was brought to room temperature and stirred for $30 \mathrm{~min}$. thereafter refluxed at $80^{\circ} \mathrm{C}$ for $4 \mathrm{~h}$. After the completion, the reaction mixture was brought to room temperature and slowly poured into cold water with continuous stirring. It was acidified (pH 3-4) with $1.5 \mathrm{~N} \mathrm{HCl}$ and was extracted with DCM $(2 \times 100 \mathrm{~mL})$, dried with anhydrous $\mathrm{Na}_{2} \mathrm{SO}_{4}$ and concentrated under reduced pressure. The products were purified using column chromatography.

1f Rf 0.8 (DCM:MeOH 9:1 v/v) Yellow solid; Yield 15\%; 1H NMR: (300 MHz, DMSO) NMR: $\delta$ 1.25-1.30 (t, J = 6 $\mathrm{Hz}, 3 \mathrm{H}, \mathrm{CH} 3), 2.68$ (s, 3H, SMe), 4.23-4.30 (q, J =6Hz, 2H, CH2, 7.10 (s, 1H, pyranone-H), 7.39-7.45 (m, 2H, ArH), 8.05-8.10 (m, 2H, ArH).

1b Rf 0.3 (DCM:MeOH 9:1 v/v) Yellow powder; Yield: 54\%; ${ }^{1} \mathrm{H}$ NMR: (400 MHz, DMSO-d 6 ): $\delta 2.68(\mathrm{~s}, 3 \mathrm{H}$, $\mathrm{SMe}), 7.13$ (s, 1H, pyranone), 7.40-7.45 (m, 2H, ArH), 8.02-8.10 (m, 2H, ArH), 12.50 (bs, 1H, COOH). 


\section{SUPPORTING INFORMATION}<smiles>[R16]C(NC(=O)c1c(S(C)(C)C)cc([Te]C(C)C)oc1=O)C(=O)OC</smiles>

Scheme S2: Preparation of Pyranone carboxamides 2a-e.

i) Glycine/alanine ester, HATU, DIPEA, DCM, rt, 3-4 h

General Procedure: To a solution of appropriate 1a-e $(2.02 \mathrm{mmol})$ in $10 \mathrm{ml}$ DCM at room temperature (rt), HATU $(2.05 \mathrm{mmol})$ was added and stirred for $10 \mathrm{~min}$. DIPEA $(7.6 \mathrm{mmol})$ was added and stirred for another $15 \mathrm{~min}$. Thereafter, Glycine/alanine ester $(2.05 \mathrm{mmol})$ was added and stirred for further $2 \mathrm{~h}$ at $\mathrm{rt}$. The reaction was monitored by TLC for completion and then was washed with water and brine subsequently. DCM was evaporated and the crude was purified by column chromatography by eluting with ethylacetate:hexane mixture to obtain 2 a-e with $>80 \%$ yield.

\section{2a, $\mathbf{A r}=\mathbf{P h}, \mathbf{R}_{1}=\mathbf{H}$}

Yellow crystalline powder; Yield $88 \%$; mp: $175-176{ }^{\circ} \mathrm{C}$; IR $(\mathrm{KBr})(v, \mathrm{~cm}-1) 3310(\mathrm{~N}-\mathrm{H}), 1744,1686$ and $1639(\mathrm{C}=\mathrm{O})$; UV $(\mathrm{MeOH}) \lambda \max =252.7 \mathrm{~nm}, 336.8 \mathrm{~nm}$; MS-ESI $(\mathrm{m} / \mathrm{z})$ : $[\mathrm{M}+]=333.9 ; 1 \mathrm{H}$ NMR: $\left(400 \mathrm{MHz}, \mathrm{DMSO}_{6} \mathrm{~d}_{6}\right) \mathrm{NMR}: \delta$ $2.60(\mathrm{~s}, 3 \mathrm{H}, \mathrm{SMe}), 3.66\left(\mathrm{~s}, 3 \mathrm{H}, \mathrm{OCH}_{3}\right), 4.06-4.07(\mathrm{~d}, J=$ $\left.6.0 \mathrm{~Hz}, 2 \mathrm{H}, \mathrm{NCH}_{2}\right), 7.18(\mathrm{~s}, 1 \mathrm{H}$, pyranone- $H), 7.59-7.62(\mathrm{~m}$, $3 \mathrm{H}, \operatorname{Ar} H), 8.02-8.05(\mathrm{~m}, 2 \mathrm{H}, \operatorname{Ar} H), 9.23-9.26(\mathrm{t}, J=6.0 \mathrm{~Hz}$, $1 \mathrm{H}, \mathrm{N} H$ amide); Elemental analysis: $\mathrm{C}: 57.73, \mathrm{H}: 4.62, \mathrm{~N}$ : 4.13, S: 9.43 .

\section{2b, $\mathrm{Ar}=4-\mathrm{F}-\mathrm{Ph}, \mathrm{R}_{\mathbf{1}}=\mathrm{CH}_{3}$}

Yellow crystalline powder; Yield 91\%; mp:187-190 ${ }^{\circ} \mathrm{C}$; IR $(\mathrm{KBr})(v, \mathrm{~cm}-1): 3315(\mathrm{~N}-\mathrm{H}), 1744,1689$ and $1649(\mathrm{C}=\mathrm{O})$; MS-ESI $(\mathrm{m} / \mathrm{z}):[\mathrm{M}+]=365.56$; $1 \mathrm{H}$ NMR: $(300 \mathrm{MHz}$, $\left.\mathrm{CDCl}_{3}\right) \mathrm{NMR}: \delta 1.50-1.52\left(\mathrm{~d}, J=6 \mathrm{~Hz}, 3 \mathrm{H}, \mathrm{CH}_{3}\right), 2.52(\mathrm{~s}$, $3 \mathrm{H}, \mathrm{SMe}), 3.76\left(\mathrm{~s}, 3 \mathrm{H}, \mathrm{OCH}_{3}\right), 4.69-4.73(\mathrm{~m}, 1 \mathrm{H}, \mathrm{NCH})$, $6.84(\mathrm{~s}, 1 \mathrm{H}$, pyranone- $H), 7.21-7.23(\mathrm{~m}, 2 \mathrm{H}, \mathrm{Ar} H), 7.87-7.92$ $(\mathrm{m}, 2 \mathrm{H}, \mathrm{Ar} H), 9.46-9.48(\mathrm{~d}, J=6 \mathrm{~Hz}, 1 \mathrm{H}, \mathrm{N} H$ amide).

\section{$\mathbf{2 c}, \mathbf{A r}=\mathbf{4 - C l - P h}, \mathbf{R}_{1}=\mathbf{H}$}

Yellow crystalline powder; Yield 86\%; mp: $185-188{ }^{\circ} \mathrm{C}$; IR $(\mathrm{KBr})(v, \mathrm{~cm}-1) 3314(\mathrm{~N}-\mathrm{H}), 1744,1690$ and $1639(\mathrm{C}=\mathrm{O})$; MS-ESI $(\mathrm{m} / \mathrm{z}):[\mathrm{M}+]=367.98 ; 1 \mathrm{H}$ NMR: $(400 \mathrm{MHz}$, $\mathrm{CDCl}_{3}$ ) NMR: $\delta 2.55(\mathrm{~s}, 3 \mathrm{H}, \mathrm{SMe}), 3.79\left(\mathrm{~s}, 3 \mathrm{H}, \mathrm{OCH}_{3}\right)$, 4.21-4.22 (d, $\left.J=4.0 \mathrm{~Hz}, 2 \mathrm{H}, \mathrm{NCH}_{2}\right), 6.48(\mathrm{~s}, 1 \mathrm{H}$, pyranoneH), 7.45-7.55 (m, 2H, ArH), 7.70-7.82 (m, 2H, ArH), 9.73 (bs, $1 \mathrm{H}, \mathrm{NH}$ amide).

\section{2d, $\mathrm{Ar}=\mathbf{4 - B r - P h ,} \mathrm{R}_{\mathbf{1}}=\mathrm{H}$}

Yellow crystalline powder; Yield 85\%; mp:186-189 ${ }^{\circ} \mathrm{C}$; IR (KBr) $(v, \mathrm{~cm}-1) 3306(\mathrm{~N}-\mathrm{H}), 1736,1697$ and $1628(\mathrm{C}=\mathrm{O})$; MS-ESI $(\mathrm{m} / \mathrm{z}):[\mathrm{M}+]=412.85$; $1 \mathrm{H}$ NMR: $\left(400 \mathrm{MHz}, \mathrm{CDCl}_{3}\right)$ NMR: $\delta 2.61$ (s, 3H, SMe), $3.78(\mathrm{~s}$, $\left.3 \mathrm{H}, \mathrm{OCH}_{3}\right), 4.10-4.12\left(\mathrm{~d}, J=5.6 \mathrm{~Hz}, 2 \mathrm{H}, \mathrm{NCH}_{2}\right), 6.61$ $(\mathrm{s}, 1 \mathrm{H}$, pyranone- $H), 7.60-7.63(\mathrm{~m}, 2 \mathrm{H}, \operatorname{Ar} H), 7.71-$ $7.73(\mathrm{~m}, 2 \mathrm{H}, \mathrm{Ar} H), 9.68-9.71(\mathrm{t}, J=5.6 \mathrm{~Hz}, 1 \mathrm{H}, \mathrm{N} H$ amide)

\section{$2 \mathrm{e}, \mathrm{Ar}=$ furan-2yl, $\mathrm{R}_{\mathbf{1}}=\mathrm{CH}_{3}$}

Yellow crystalline powder; Yield 85\%; mp:176-178 ${ }^{\circ} \mathrm{C}$; IR $(\mathrm{KBr})(\mathrm{v}, \mathrm{cm}-1) 3306(\mathrm{~N}-\mathrm{H}), 1725,1686$ and $1635(\mathrm{C}=\mathrm{O})$; MS-ESI $(\mathrm{m} / \mathrm{z}):[\mathrm{M}+]=337.8$; $1 \mathrm{H}$ NMR: $\left(400 \mathrm{MHz}, \mathrm{CDCl}_{3}\right) \mathrm{NMR}: \delta 1.41-1.43(\mathrm{~d}, J=8 \mathrm{~Hz}, 3 \mathrm{H}$, $\left.\mathrm{CH}_{3}\right), 2.56(\mathrm{~s}, 3 \mathrm{H}, \mathrm{SMe}), 3.69$ (s, 3H, $\left.\mathrm{OCH}_{3}\right), 4.50-4.52$ $(\mathrm{m}, 1 \mathrm{H}, \mathrm{NCH}), 6.36(\mathrm{~s}, 1 \mathrm{H}$, pyranone- $H), 7.03-7.48(\mathrm{~m}$, $3 \mathrm{H}, \mathrm{Ar}-H), 9.71$ (bs, $1 \mathrm{H}, \mathrm{NH}$ amide).

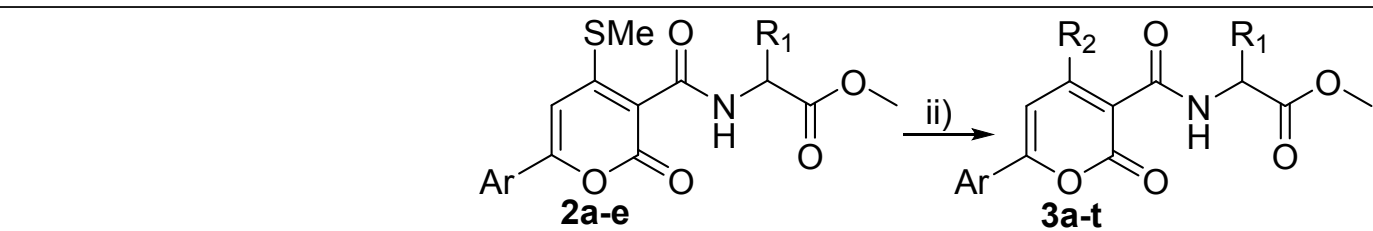

Scheme S3: Preparation of compounds 3a-t.

ii) $\mathrm{R}_{2} \mathrm{NH}_{2}$, Ethanol, reflux, 2-6h.

General Procedure: To a solution of appropriate 2a-e $(1.5 \mathrm{mmol})$ in ethanol, appropriate amine $\left(\mathrm{RNH}_{2}, 2.2 \mathrm{mmol}\right)$ was added and refluxed for $2 \mathrm{~h}$ at $80^{\circ} \mathrm{C}$. The completion of the reaction was monitored by TLC. Ethanol was removed under reduced pressure. The crude was dissolved in DCM, washed with water, brine and the organic layer was concentrated under reduced pressure. The crude was purified using column chromatography by eluting with a mixture of hexane and ethylacetate to get $\mathbf{3 a - t}$ in $40-65 \%$ yield.

3a, $\mathrm{Ar}=\mathrm{Ph}, \mathrm{R}_{1}=\mathrm{H}, \mathrm{R}_{2}=$ isopentylamino

White powder; Yield $54 \%$; mp: $96-100{ }^{\circ} \mathrm{C}$; IR $(\mathrm{KBr})\left(\mathrm{v}\right.$, White powder; Yield $62 \%$; mp: $170{ }^{\circ} \mathrm{C}$; IR $(\mathrm{KBr})\left(\mathrm{v}, \mathrm{cm}^{-}\right.$ 


\section{SUPPORTING INFORMATION}

$\left.\mathrm{cm}^{-1}\right): \quad 1735, \quad 1682 \quad(\mathrm{C}=\mathrm{O} \quad$ stretching $), \quad 3285 \quad(\mathrm{~N}-\mathrm{H}$ stretching), $3081(\mathrm{C}-\mathrm{H})$; MS-ESI $(\mathrm{m} / \mathrm{z}):\left[\mathrm{M}^{+}\right] 372.7 ;{ }^{1} \mathrm{H}$ NMR $\left(400 \mathrm{MHz}, \mathrm{CDCl}_{3}\right)$ NMR: $\delta 0.97-0.99(\mathrm{~d}, J=6.8 \mathrm{~Hz}$, $\left.6 \mathrm{H}, \mathrm{CH}\left(\mathrm{CH}_{3}\right)_{2}\right), 1.58-1.64\left(\mathrm{~m}, 2 \mathrm{H}, \mathrm{CH}_{2}\right), 1.74-1.77(\mathrm{~m}$, $\left.1 \mathrm{H}, \mathrm{CH}\left(\mathrm{CH}_{3}\right)_{2}\right), 3.36-3.41\left(\mathrm{~m}, 2 \mathrm{H}, \mathrm{N}-\mathrm{CH}_{2}\right), 3.77(\mathrm{~s}, 3 \mathrm{H}$, O-CH $H_{3}, 4.13-4.15\left(\mathrm{~d}, J=5.6 \mathrm{~Hz}, 2 \mathrm{H}, \mathrm{CH}_{2}\right), 6.50(\mathrm{~s}, 1 \mathrm{H}$, pyranone- $H$ ), 7.47-7.52 (m, 3H, Ar- $H), 7.85-7.87(\mathrm{~m}, 2 \mathrm{H}$, Ar- $H$ ), 9.80 (bs, $1 \mathrm{H}, \mathrm{CON} H), 11.15$ (bs, $1 \mathrm{H}, \mathrm{N} H)$.

\section{$\mathbf{3 b}, \mathbf{A r}=\mathbf{P h}, \mathbf{R}_{\mathbf{1}}=\mathbf{H}, \mathbf{R}_{\mathbf{2}}=$ allylamino}

White powder; Yield $56 \%$; mp: $140^{\circ} \mathrm{C}$; IR $(\mathrm{KBr})\left(v, \mathrm{~cm}^{-1}\right)$ : 1739, 1689 ( $\mathrm{C}=\mathrm{O}$ stretching), 3267 ( $\mathrm{N}-\mathrm{H}$ stretching), 3082 (C-H); MS-ESI $(\mathrm{m} / \mathrm{z}):\left[\mathrm{M}^{+}\right]$342.6; ${ }^{1} \mathrm{H}$ NMR $(400 \mathrm{MHz}$, $\left.\mathrm{CDCl}_{3}\right)$ NMR: $\delta 3.78\left(\mathrm{~s}, 3 \mathrm{H}, \mathrm{O}-\mathrm{CH}_{3}\right), 4.03-4.06(\mathrm{~m}, 2 \mathrm{H}$, $\left.\mathrm{CH}_{2}\right), 4.14-4.15\left(\mathrm{~d}, \mathrm{~J}=6 \mathrm{~Hz}, 2 \mathrm{H}, \mathrm{CH}_{2}\right), 5.28-5.35(\mathrm{~m}, 2 \mathrm{H}$, $\left.\mathrm{CH}_{2}\right), 5.92-5.96(\mathrm{~m}, 1 \mathrm{H}, \mathrm{CH}), 6.48(\mathrm{~s}, 1 \mathrm{H}$, pyranone- $H)$, 7.46-7.52 (m, 3H, Ar- $H$ ), 7.82-7.85 (m, 2H, Ar- H), 9.80 (bs, 1H, CONH), 11.33 (bs, 1H, NH).

3c, $\mathbf{A r}=\mathbf{P h}, \mathbf{R}_{1}=\mathbf{H}, \mathbf{R}_{2}=$ cyclopropylamino

White powder; Yield $64 \%$; mp: $200^{\circ} \mathrm{C}$; IR $(\mathrm{KBr})\left(v, \mathrm{~cm}^{-1}\right)$ : 1731, $1686(\mathrm{C}=\mathrm{O}$ stretching), 3237 ( $\mathrm{N}-\mathrm{H}$ stretching), 3073 $(\mathrm{C}-\mathrm{H})$; MS-ESI $(\mathrm{m} / \mathrm{z})$ : $\left[\mathrm{M}^{+}\right]$342.6; ${ }^{1} \mathrm{H}$ NMR $(400 \mathrm{MHz}$, $\left.\mathrm{CDCl}_{3}\right)$ NMR: 0.74-0.78 (m, 2H, $\left.\mathrm{CH}_{2}\right), 0.93-0.98(\mathrm{~m}, 2 \mathrm{H}$, $\left.\mathrm{CH}_{2}\right), 2.67-2.72(\mathrm{~m}, 1 \mathrm{H}, \mathrm{N}-\mathrm{CH}), 3.77\left(\mathrm{~s}, 3 \mathrm{H}, \mathrm{O}-\mathrm{CH}_{3}\right)$, 4.11-4.12 $\left(\mathrm{d}, J=5.6 \mathrm{~Hz}, 2 \mathrm{H}, \mathrm{CH}_{2}\right), 6.94(\mathrm{~s}, 1 \mathrm{H}$, pyranone$H)$, 7.47-7.53 (m, 3H, Ar- $H$ ), 7.87-7.90 (m, $2 \mathrm{H}$, Ar- $H$ ), 9.74-9.77 (t, $J=5.6 \mathrm{~Hz} 1 \mathrm{H}, \mathrm{CON} H), 11.02$ (bs, 1H, NH).

\section{3d, $\mathrm{Ar}=\mathbf{4 - F - P h}, \mathrm{R}_{\mathbf{1}}=\mathrm{CH}_{\mathbf{3}}, \mathrm{R}_{\mathbf{2}}=$ isopentylamino}

White powder; Yield $63 \%$; mp: $70-73^{\circ} \mathrm{C}$; IR $(\mathrm{KBr})\left(v, \mathrm{~cm}^{-}\right.$ $\left.{ }^{1}\right): 1744,1682(\mathrm{C}=\mathrm{O}$ stretching $), 3267(\mathrm{~N}-\mathrm{H}$ stretching $)$, $3075(\mathrm{C}-\mathrm{H})$; UV $(\mathrm{MeOH}) \lambda \max =222.2 \mathrm{~nm}, 244.7 \mathrm{~nm}$, $304.3 \mathrm{~nm}$; MS-ESI $(\mathrm{m} / \mathrm{z}):\left[\mathrm{M}^{+}+1\right]$ 405.17; ${ }^{1} \mathrm{H}$ NMR (300 $\mathrm{MHz}, \mathrm{CDCl}_{3}$ ) NMR: $\delta$ 0.96-0.98 (d, $J=6 \mathrm{~Hz}, 6 \mathrm{H}$, $\left.\mathrm{CH}\left(\mathrm{CH}_{3}\right)_{2}\right), 1.49-1.52\left(\mathrm{~d}, J=9 \mathrm{~Hz}, 3 \mathrm{H}, \mathrm{CH}_{3}\right), 1.57-1.64$ $\left(\mathrm{m}, 2 \mathrm{H}, \mathrm{CH}_{2}\right), 1.72-1.76\left(\mathrm{~m}, 1 \mathrm{H}, \mathrm{CH}\left(\mathrm{CH}_{3}\right)\right), 3.33-3.40(\mathrm{~m}$, $\left.2 \mathrm{H}, \mathrm{N}-\mathrm{CH}_{2}\right), 3.76\left(\mathrm{~s}, 3 \mathrm{H}, \mathrm{O}-\mathrm{CH}_{3}\right), 4.58-4.63(\mathrm{~m}, 1 \mathrm{H}, \mathrm{CH})$, $6.42(\mathrm{~s}, 1 \mathrm{H}$, pyranone- $H), 7.14-7.20(\mathrm{~m}, 2 \mathrm{H}, \mathrm{Ar}-H), 7.83-$ $7.88(\mathrm{~m}, 2 \mathrm{H}, \mathrm{Ar}-H), 9.71-9.73(\mathrm{~d}, J=6 \mathrm{~Hz}, 1 \mathrm{H}, \mathrm{CON} H)$, 11.18 (bs, $1 \mathrm{H}, \mathrm{N} H) ;{ }^{13} \mathrm{CNMR}\left(75 \mathrm{MHz}, \mathrm{CDCl}_{3}\right)$ NMR: $17.99,22.41,25.77,38.17,41.49,47.90,52.35,85.61$, $92.33,116.08,116.37,127.30,128.36,128.48,159.67$, $160.84,163.58,168.88,173.57$.

\section{$\mathbf{3 e}, \mathbf{A r}=\mathbf{4 - F - P h}, \mathbf{R}_{1}=\mathbf{C H}_{3}, \mathbf{R}_{\mathbf{2}}=$ allylamino}

White powder; Yield $64 \%$; mp: 80-88 ${ }^{\circ} \mathrm{C}$; IR $(\mathrm{KBr})\left(\mathrm{v}, \mathrm{cm}^{-}\right.$ $\left.{ }^{1}\right): 1736,1682,1648 \quad(\mathrm{C}=\mathrm{O}$ stretching $), 3248 \quad(\mathrm{~N}-\mathrm{H}$ stretching), 3094 (C-H); MS-ESI (m/z): $\left[\mathrm{M}^{+}+1\right] 375.12 ;{ }^{1} \mathrm{H}$ NMR $\left(400 \mathrm{MHz}, \mathrm{CDCl}_{3}\right)$ NMR: $\delta 1.43-1.45(\mathrm{~d}, \mathrm{~J}=8 \mathrm{~Hz}$, $\left.3 \mathrm{H}, \mathrm{CH}_{3}\right), 3.67\left(\mathrm{~s}, 3 \mathrm{H}, \mathrm{O}-\mathrm{CH}_{3}\right), 3.93-3.96\left(\mathrm{~m}, 2 \mathrm{H}, \mathrm{CH}_{2}\right)$, 4.52-4.61 (m, $1 \mathrm{H}, \mathrm{CH}), 5.19-5.25\left(\mathrm{~m}, 2 \mathrm{H}, \mathrm{CH}_{2}\right), 5.79-$ $5.91(\mathrm{~m}, 1 \mathrm{H}, \mathrm{CH}), 6.86(\mathrm{~s}, 1 \mathrm{H}$, pyranone- $H), 7.86-7.90(\mathrm{~m}$, $2 \mathrm{H}, \operatorname{Ar}-H), 7.15-7.21(\mathrm{~m}, 2 \mathrm{H}, \operatorname{Ar}-H), 9.67-9.69(\mathrm{~d}, J=$ $6 \mathrm{~Hz}, 1 \mathrm{H}, \mathrm{CON} H), 11.06$ (s, $1 \mathrm{H}, \mathrm{N} H)$.

\section{3f, $\mathrm{Ar}=4-\mathrm{F}-\mathrm{Ph}, \mathrm{R}_{\mathbf{1}}=\mathrm{CH}_{\mathbf{3}}, \mathrm{R}_{\mathbf{2}}=$ cyclopropylamino}

White powder; Yield $66 \%$; mp: $142-146{ }^{\circ} \mathrm{C}$; IR $(\mathrm{KBr})(v$, $\left.\mathrm{cm}^{-1}\right): \quad 1740, \quad 1690 \quad(\mathrm{C}=\mathrm{O}$ stretching $), \quad 3267 \quad(\mathrm{~N}-\mathrm{H}$ stretching), $3082(\mathrm{C}-\mathrm{H})$; UV $(\mathrm{MeOH}) \lambda \max =222.4 \mathrm{~nm}$, $244.7 \mathrm{~nm}, 304.3 \mathrm{~nm}$; MS-ESI $(\mathrm{m} / \mathrm{z}):\left[\mathrm{M}^{+}+1\right] 375.13 ;{ }^{1} \mathrm{H}$ $\left.{ }^{1}\right)$ : 1739, 1685 ( $\mathrm{C}=\mathrm{O}$ stretching), 3268 (N-H stretching), $3074(\mathrm{C}-\mathrm{H})$; MS-ESI $(\mathrm{m} / \mathrm{z}):\left[\mathrm{M}^{+}\right]$376.9; ${ }^{1} \mathrm{H}$ NMR (400 $\left.\mathrm{MHz}, \mathrm{CDCl}_{3}\right) \quad \mathrm{NMR}: \delta$ 0.74-0.78 $\left(\mathrm{m}, 2 \mathrm{H}, \mathrm{CH}_{2}\right), 0.93-$ $0.98\left(\mathrm{~m}, 2 \mathrm{H}, \mathrm{CH}_{2}\right), 2.69-2.70(\mathrm{~m}, 1 \mathrm{H}, \mathrm{N}-\mathrm{CH}), 3.77(\mathrm{~s}$, $\left.3 \mathrm{H}, \mathrm{O}-\mathrm{CH}_{3}\right), 4.10-4.12\left(\mathrm{~d}, \mathrm{~J}=6 \mathrm{~Hz}, 2 \mathrm{H}, \mathrm{CH}_{2}\right), 6.90(\mathrm{~s}$, $1 \mathrm{H}$, pyranone- $H)$, 7.27-7.48 (m, 2H, Ar- $H), 7.80-7.83(\mathrm{~m}$, 2H, Ar- $H$ ), 9.69-9.72 (t, $J=5.6 \mathrm{~Hz}, 1 \mathrm{H}, \mathrm{CON} H), 11.056$ (bs, $1 \mathrm{H}, \mathrm{N} H$ ).

3l, $\mathbf{A r}=4-\mathrm{Cl}-\mathrm{Ph}, \mathrm{R}_{\mathbf{1}}=\mathrm{H}, \mathrm{R}_{\mathbf{2}}=$ cyclohexylamino White powder; Yield $39 \%$; mp: $182-188^{\circ} \mathrm{C}$; IR $(\mathrm{KBr})(v$, $\left.\mathrm{cm}^{-1}\right): \quad 1749, \quad 1685 \quad(\mathrm{C}=\mathrm{O}$ stretching $), 3317 \quad(\mathrm{~N}-\mathrm{H}$ stretching), 3042 (C-H); MS-ESI (m/z): $\left[\mathrm{M}^{+}\right]$418.9; ${ }^{1} \mathrm{H}$ NMR $\left(400 \mathrm{MHz}, \mathrm{CDCl}_{3}\right)$ NMR: $\delta 1.31-2.01(\mathrm{~m}, 10 \mathrm{H}$, cyclohexyl), 3.50-3.55 (m, 1H, N-CH), $3.77(\mathrm{~s}, 3 \mathrm{H}, \mathrm{O}-$ $\left.\mathrm{CH}_{3}\right), 4.15-4.17\left(\mathrm{~d}, J=5.6 \mathrm{~Hz}, 2 \mathrm{H}, \mathrm{CH}_{2}\right), 6.46(\mathrm{~s}, 1 \mathrm{H}$, pyranone- $H), 7.46-7.48(\mathrm{~m}, 2 \mathrm{H}, \mathrm{Ar}-H), 7.78-7.80(\mathrm{~m}, 2 \mathrm{H}$, Ar- $H), 9.79$ (s, 1H, CONH), 11.33-11.35 (bs, $1 \mathrm{H}, \mathrm{N} H)$.

\section{$\mathbf{3 m}, \mathbf{A r}=\mathbf{4 - C l - P h}, \mathbf{R}_{\mathbf{1}}=\mathbf{H}, \mathbf{R}_{\mathbf{2}}=$ isopropylamino}

White powder; Yield $52 \%$; mp: $181-186^{\circ} \mathrm{C}$; IR $(\mathrm{KBr})(v$, $\left.\mathrm{cm}^{-1}\right): \quad 1742, \quad 1683 \quad(\mathrm{C}=\mathrm{O}$ stretching $), 3267 \quad(\mathrm{~N}-\mathrm{H}$ stretching), $3081(\mathrm{C}-\mathrm{H}) ;$ MS-ESI $(\mathrm{m} / \mathrm{z}):\left[\mathrm{M}^{+}\right] 378.9 ;{ }^{1} \mathrm{H}$ NMR $\left(400 \mathrm{MHz}, \mathrm{CDCl}_{3}\right)$ NMR: $\delta$ 1.34-1.36 $(\mathrm{d}, J=$ $\left.6.8 \mathrm{~Hz}, 6 \mathrm{H},\left(\mathrm{CH}_{3}\right)_{2}\right), 3.77\left(\mathrm{~s}, 3 \mathrm{H}, \mathrm{O}-\mathrm{CH}_{3}\right), 3.86-3.90(\mathrm{~m}$, $\left.1 \mathrm{H}, \mathrm{CH}\left(\mathrm{CH}_{3}\right)_{3}\right), 4.13-4.14\left(\mathrm{~d}, J=5.6 \mathrm{~Hz}, 2 \mathrm{H}, \mathrm{CH}_{2}\right) 6.47$ (s, $1 \mathrm{H}$, pyranone- $H)$, 7.44-7.47 (m, $2 \mathrm{H}, \mathrm{Ar} H), 7.77-7.80$ $(\mathrm{m}, 2 \mathrm{H}, \operatorname{Ar} H), 9.76-9.79(\mathrm{t}, J=5.2 \mathrm{~Hz}, 1 \mathrm{H}, \mathrm{CON} H)$, $11.22-11.23(\mathrm{~d}, J=6 \mathrm{~Hz}, 1 \mathrm{H}, \mathrm{N} H)$.

\section{$\mathbf{3 n}, \mathbf{A r}=\mathbf{4 - B r}-\mathrm{Ph}, \mathrm{R}_{\mathbf{1}}=\mathbf{H}, \mathrm{R}_{2}=$ isopentylamino}

White powder; Yield $61 \%$; mp: $141-148{ }^{\circ} \mathrm{C}$; IR (KBr) $(v$, $\left.\mathrm{cm}^{-1}\right): \quad 1739, \quad 1689 \quad(\mathrm{C}=\mathrm{O}$ stretching $), 3267 \quad(\mathrm{~N}-\mathrm{H}$ stretching), $3082(\mathrm{C}-\mathrm{H})$; MS-ESI (m/z): $\left[\mathrm{M}^{+}+1\right]$ 452.9; ${ }^{1} \mathrm{H}$ NMR $\left(400 \mathrm{MHz}, \mathrm{CDCl}_{3}\right)$ NMR: $\delta$ 0.96-0.98 $(\mathrm{d}, J=$ $\left.6.4 \mathrm{~Hz}, 6 \mathrm{H}, \mathrm{CH}\left(\mathrm{CH}_{3}\right)_{2}\right), 1.58-1.63\left(\mathrm{~m}, 2 \mathrm{H}, \mathrm{CH}_{2}\right), 1.73-$ $1.76\left(\mathrm{~m}, 1 \mathrm{H}, \mathrm{CH}\left(\mathrm{CH}_{3}\right)_{2}\right), 3.35-3.38\left(\mathrm{~m}, 2 \mathrm{H}, \mathrm{N}-\mathrm{CH}_{2}\right), 3.77$ $\left(\mathrm{s}, 3 \mathrm{H}, \mathrm{O}-\mathrm{CH}_{3}\right), 4.12-4.14\left(\mathrm{~d}, J=5.6 \mathrm{~Hz}, 2 \mathrm{H}, \mathrm{CH}_{2}\right), 6.47$ (s, 1H, pyranone- $H)$, 7.60-7.63 (m, 2H, Ar- $H), 7.71-7.73$ $(\mathrm{m}, 2 \mathrm{H}, \mathrm{Ar}-H), 9.72-9.73$ (bs, $1 \mathrm{H}, \mathrm{CON} H), 11.18$ (bs, $1 \mathrm{H}, \mathrm{N} H)$.

\section{3o, $\mathrm{Ar}=4-\mathrm{Br}-\mathrm{Ph}, \mathrm{R}_{\mathbf{1}}=\mathrm{H}, \mathrm{R}_{\mathbf{2}}=$ allylamino}

White powder; Yield $64 \%$; mp: $138-145^{\circ} \mathrm{C}$; IR $(\mathrm{KBr})(v$, $\left.\mathrm{cm}^{-1}\right): 1759,1684,1640(\mathrm{C}=\mathrm{O}$ Stretching $), 3335(\mathrm{~N}-\mathrm{H}$ Stretching); MS-ESI $(\mathrm{m} / \mathrm{z}):\left[\mathrm{M}^{+}\right] 420.9 ;{ }^{1} \mathrm{H}$ NMR (400 $\mathrm{MHz}, \mathrm{CDCl}_{3}$ ) NMR: $\delta 3.77\left(\mathrm{~s}, 3 \mathrm{H}, \mathrm{O}-\mathrm{CH}_{3}\right), 4.02-4.05$ $\left(\mathrm{m}, 2 \mathrm{H}, \mathrm{CH}_{2}\right), 4.13-4.14\left(\mathrm{~d}, \mathrm{~J}=5.6 \mathrm{~Hz}, 2 \mathrm{H}, \mathrm{CH}_{2}\right), 5.27-$ $5.34\left(\mathrm{~m}, 2 \mathrm{H}, \mathrm{CH}_{2}\right), 5.87-5.95(\mathrm{~m}, 1 \mathrm{H}, \mathrm{CH}), 6.45(\mathrm{~s}, 1 \mathrm{H}$, pyranone- $H), 7.60-7.62(\mathrm{~m}, 2 \mathrm{H}, \mathrm{Ar}-H), 7.68-7.70(\mathrm{~m}, 2 \mathrm{H}$, Ar- $H$ ), 9.72-9.75 (t, $J=5.6 \mathrm{~Hz}, 1 \mathrm{H}, \mathrm{CON} H), 11.35$ (bs, $1 \mathrm{H}, \mathrm{N} H)$.

\section{$\mathbf{3 p}, \mathbf{A r}=\mathbf{4}-\mathrm{Br}-\mathrm{Ph}, \mathrm{R}_{\mathbf{1}}=\mathbf{H}, \mathrm{R}_{\mathbf{2}}=$ cycloproylamino}

White powder; Yield $65 \%$; mp: $180-183{ }^{\circ} \mathrm{C}$; IR $(\mathrm{KBr})(v$, $\left.\mathrm{cm}^{-1}\right): 1759,1682,1639(\mathrm{C}=\mathrm{O}$ Stretching $), 3333(\mathrm{~N}-\mathrm{H}$ Stretching) $3078(\mathrm{C}-\mathrm{H})$; UV $(\mathrm{MeOH}) \lambda \max =227.9 \mathrm{~nm}$, $248.3 \mathrm{~nm}, 311.7 \mathrm{~nm} ;$ MS-ESI $(\mathrm{m} / \mathrm{z}):\left[\mathrm{M}^{+}+1\right] 422.8 ;{ }^{1} \mathrm{H}$ 
NMR (300 MHz, $\left.\mathrm{CDCl}_{3}\right)$ NMR: 0.74-0.76 (m, 2H, $\left.\mathrm{CH}_{2}\right)$, 0.93-0.95 (m, 2H, $\left.\mathrm{CH}_{2}\right), 1.49-1.51\left(\mathrm{~d}, J=6 \mathrm{~Hz}, 3 \mathrm{H}, \mathrm{CH}_{3}\right)$, 2.65-2.70 (m, $1 \mathrm{H}, \quad \mathrm{CH})$, $3.76\left(\mathrm{~s}, 3 \mathrm{H}, \mathrm{O}-\mathrm{CH}_{3}\right), 4.52-4.61(\mathrm{~m}, 1 \mathrm{H}, \mathrm{CH}), 6.86(\mathrm{~s}, 1 \mathrm{H}$, pyranone- $H), 7.86-7.90(\mathrm{~m}, 2 \mathrm{H}, \mathrm{Ar}-H), 7.15-7.21(\mathrm{~m}, 2 \mathrm{H}$, Ar- $H), 9.67-9.69(\mathrm{~d}, J=6 \mathrm{~Hz}, 1 \mathrm{H}, \mathrm{CON} H), 11.06(\mathrm{~s}, 1 \mathrm{H}$, $\mathrm{N} H$ ).

\section{$\mathbf{3 g}, \mathbf{A r}=\mathbf{4 - F}-\mathrm{Ph}, \mathrm{R}_{\mathbf{1}}=\mathrm{CH}_{\mathbf{3}}, \mathrm{R}_{\mathbf{2}}=$ cyclohexylamino}

White powder; Yield $40 \%$; mp: $94-95{ }^{\circ} \mathrm{C}$; IR $(\mathrm{KBr})\left(v, \mathrm{~cm}^{-}\right.$ $\left.{ }^{1}\right)$ : 1744, $1674(\mathrm{C}=\mathrm{O}$ stretching), $3414(\mathrm{~N}-\mathrm{H}$ stretching), 2978 (C-H); MS-ESI (m/z): $\left[\mathrm{M}^{+}+1\right]$ 417.93; ${ }^{1} \mathrm{H}$ NMR (400 $\left.\mathrm{MHz}, \mathrm{CDCl}_{3}\right)$ NMR: $\delta$ 1.24-2.04 $(\mathrm{m}, 10 \mathrm{H}$, cyclohexyl $\left.\mathrm{CH}_{2}\right), 1.42-1.44\left(\mathrm{~d}, \mathrm{~J}=8 \mathrm{~Hz}, 3 \mathrm{H}, \mathrm{CH}_{3}\right) 3.51-3.55(\mathrm{~m}, 1 \mathrm{H}$, $\mathrm{CH}-\mathrm{N}), 3.69\left(\mathrm{~s}, 3 \mathrm{H}, \mathrm{O}-\mathrm{CH}_{3}\right), 4.53(\mathrm{~m}, 1 \mathrm{H}, \mathrm{CH}), 6.36(\mathrm{~s}$, $1 \mathrm{H}$, pyranone- $H)$, 7.86-7.90 (m, 2H, Ar- $H), 7.15-7.21(\mathrm{~m}$, 2H, Ar- $H$ ), 9.71 (bs, 1H, CONH), 11.18 (bs, 1H, NH).

\section{3h, Ar $=4-F-P h, ~ R_{1}=\mathbf{C H}_{3}, \mathbf{R}_{2}=$ pyrrolidinyl}

White powder; Yield $41 \%$; mp: $134-140{ }^{\circ} \mathrm{C}$; IR (KBr) ( $v$, $\left.\mathrm{cm}^{-1}\right): 1743,1685,1623(\mathrm{C}=\mathrm{O}$ stretching $), 3329(\mathrm{~N}-\mathrm{H}$ stretching), $3013(\mathrm{C}-\mathrm{H})$ MS-ESI $(\mathrm{m} / \mathrm{z}):\left[\mathrm{M}^{+}\right] 388.91 ;{ }^{1} \mathrm{H}$ NMR $\left(400 \mathrm{MHz}, \mathrm{CDCl}_{3}\right)$ NMR: 0.78-1.56 (m, 8H, pyrolidinyl), $1.43-1.45\left(\mathrm{~d}, J=8 \mathrm{~Hz}, 3 \mathrm{H}, \mathrm{CH}_{3}\right), 3.68(\mathrm{~s}, 3 \mathrm{H}$, $\left.\mathrm{O}-\mathrm{CH}_{3}\right), 4.52-4.61(\mathrm{~m}, 1 \mathrm{H}, \mathrm{CH}), 6.41(\mathrm{~s}, 1 \mathrm{H}$, pyranone- $H)$, $7.08(\mathrm{~m}, 2 \mathrm{H}, \mathrm{Ar}-H), 7.20(\mathrm{~m}, 2 \mathrm{H}, \mathrm{Ar}-H), 9.67-9.69(\mathrm{~d}, J=$ $8 \mathrm{~Hz}, 1 \mathrm{H}, \mathrm{CON} H), 11.06(\mathrm{~s}, 1 \mathrm{H}, \mathrm{N} H)$.

\section{3i, $\mathbf{A r}=\mathbf{4 - C l - P h}, \mathrm{R}_{\mathbf{1}}=\mathbf{H}, \mathrm{R}_{\mathbf{2}}=$ isopentylamino}

White powder; Yield $60 \%$; mp: $120^{\circ} \mathrm{C}$; IR $(\mathrm{KBr})\left(\mathrm{v}, \mathrm{cm}^{-1}\right)$ : 1739, 1689 ( $\mathrm{C}=\mathrm{O}$ stretching), 3267 ( $\mathrm{N}-\mathrm{H}$ stretching), 3082 (C-H); MS-ESI (m/z): $\left[\mathrm{M}^{+}\right]$407; ${ }^{1} \mathrm{H}$ NMR $(400 \mathrm{MHz}$, $\mathrm{CDCl}_{3}$ ) NMR: $0.96-0.98\left(\mathrm{~d}, J=6.8 \mathrm{~Hz}, 6 \mathrm{H}, \mathrm{CH}\left(\mathrm{CH}_{3}\right)_{2}\right)$, 1.58-1.62 (m, 2H, $\left.\mathrm{CH}_{2}\right), 1.64-1.77\left(\mathrm{~m}, 1 \mathrm{H}, \mathrm{CH}\left(\mathrm{CH}_{3}\right)_{2}\right)$, 3.35-3.40 (m, 2H, N-CH $)_{2}, 3.77$ (s, 3H, O-CH $\left.H_{3}\right), 4.12-4.14$ $\left(\mathrm{d}, J=6 \mathrm{~Hz}, 2 \mathrm{H}, \mathrm{CH}_{2}\right), 6.47(\mathrm{~s}, 1 \mathrm{H}$, pyranone- $H), 7.45-$ 7.47 (m, 2H, Ar- $H$ ), 7.78-7.81 (m, 2H, Ar-H), 9.73-9.75 (t, $J=5.6 \mathrm{~Hz}, 1 \mathrm{H}, \mathrm{CON} H), 11.18(\mathrm{bs}, 1 \mathrm{H}, \mathrm{N} H)$

\section{$\mathbf{3 j}$, Ar $=\mathbf{4 - C l - P h , ~} \mathbf{R}_{\mathbf{1}}=\mathbf{H}, \mathbf{R}_{\mathbf{2}}=$ allylamino}

White powder; Yield $61 \%$; mp $135-140^{\circ} \mathrm{C}$; IR $(\mathrm{KBr})(v$, $\left.\mathrm{cm}^{-1}\right): \quad 1738, \quad 1689 \quad(\mathrm{C}=\mathrm{O}$ stretching $), \quad 3268 \quad(\mathrm{~N}-\mathrm{H}$ stretching), $3083(\mathrm{C}-\mathrm{H})$; MS-ESI $(\mathrm{m} / \mathrm{z}):\left[\mathrm{M}^{+}\right] 376.9 ;{ }^{1} \mathrm{H}$ NMR $\left(400 \mathrm{MHz}, \mathrm{CDCl}_{3}\right)$ NMR: $\delta 3.77\left(\mathrm{~s}, 3 \mathrm{H}, \mathrm{O}-\mathrm{CH}_{3}\right)$, 4.03-4.05 (m, $\left.2 \mathrm{H}, \mathrm{CH}_{2}\right), 4.13-4.14(\mathrm{~d}, J=5.6 \mathrm{~Hz}, 2 \mathrm{H}$, $\left.\mathrm{CH}_{2}\right)$, 5.28-5.34 (m, $\left.2 \mathrm{H}, \mathrm{CH}_{2}\right), 5.89-5.96(\mathrm{~m}, 1 \mathrm{H}, \mathrm{CH})$, $6.44(\mathrm{~s}, 1 \mathrm{H}$, pyranone- $H), 7.43-7.45(\mathrm{~m}, 2 \mathrm{H}, \mathrm{Ar}-H), 7.74-$ $7.77(\mathrm{~m}, 2 \mathrm{H}, \mathrm{Ar}-H), 9.73-9.76(\mathrm{t}, J=5.6 \mathrm{~Hz}, 1 \mathrm{H}, \mathrm{CON} H)$, 11.35 (bs, $1 \mathrm{H}, \mathrm{N} H$ )
NMR $\left(400 \mathrm{MHz}, \mathrm{CDCl}_{3}\right)$ NMR: $\delta$ 0.74-0.77 $(\mathrm{m}, 2 \mathrm{H}$, $\left.\mathrm{CH}_{2}\right)$, 0.93-0.98 (m, 2H, $\left.\mathrm{CH}_{2}\right), 2.67-2.69(\mathrm{~m}, 1 \mathrm{H}, \mathrm{CH})$, $3.77\left(\mathrm{~s}, 3 \mathrm{H}, \mathrm{O}-\mathrm{CH}_{3}\right), 4.10-4.12\left(\mathrm{~d}, J=5.6 \mathrm{~Hz}, 2 \mathrm{H}, \mathrm{CH}_{2}\right)$, $6.91(\mathrm{~s}, 1 \mathrm{H}$, pyranone- $H), 7.61-7.63(\mathrm{~m}, 2 \mathrm{H}, \mathrm{Ar}-H), 7.73-$ $7.75(\mathrm{~m}, 2 \mathrm{H}, \mathrm{Ar}-H), 9.69-9.71(\mathrm{t}, J=5.6 \mathrm{~Hz}, 1 \mathrm{H}, \mathrm{CON} H)$, 11.06 (bs, $1 \mathrm{H}, \mathrm{NH}) ;{ }^{13} \mathrm{CNMR}\left(75 \mathrm{MHz}, \mathrm{CDCl}_{3}\right.$ ) NMR: 7.73, 24.54, 41.01, 52.29, 86.52, 94.11, 126.35, 127.66, $129.86,132.28,159.45,162.74,163.42,169.22,170.40$

$\mathbf{3 q}, \mathbf{A r}=\mathbf{4 - B r}-\mathbf{P h}, \mathbf{R}_{\mathbf{1}}=\mathbf{H}, \mathbf{R}_{\mathbf{2}}=$ cyclohexylamino White powder; Yield 54\%; mp: 200-202 ${ }^{\circ} \mathrm{C}$; IR $(\mathrm{KBr})(\mathrm{v}$, $\left.\mathrm{cm}^{-1}\right): \quad 1739, \quad 1689 \quad(\mathrm{C}=\mathrm{O}$ stretching $), 3267 \quad(\mathrm{~N}-\mathrm{H}$ stretching), $3082(\mathrm{C}-\mathrm{H})$; MS-ESI (m/z): $\left[\mathrm{M}^{+}+1\right]$ 464.5; ${ }^{1} \mathrm{H}$ NMR (400 MHz, $\left.\mathrm{CDCl}_{3}\right)$ NMR: $\delta 1.24-2.04(\mathrm{~m}, 10 \mathrm{H}$, cyclohexyl $\left.\mathrm{CH}_{2}\right), 3.54(\mathrm{~m}, 1 \mathrm{H}, \mathrm{CH}-\mathrm{N}), 3.77(\mathrm{~s}, 3 \mathrm{H}, \mathrm{O}-$ $\left.\mathrm{CH}_{3}\right), 4.13-4.15\left(\mathrm{~d}, J=8 \mathrm{~Hz}, 2 \mathrm{H}, \mathrm{CH}_{2}\right), 6.45(\mathrm{~s}, 1 \mathrm{H}$, pyranone- $H), 7.61-7.62(\mathrm{~m}, 2 \mathrm{H}, \mathrm{Ar}-H), 7.70-7.72(\mathrm{~m}, 2 \mathrm{H}$, Ar- $H$ ), 9.76-9.79 (t, $J=8 \mathrm{~Hz}, 1 \mathrm{H}, \mathrm{CON} H), 11.32-11.33$ (d, $J=4 \mathrm{~Hz}, 1 \mathrm{H}, \mathrm{N} H)$.

$3 \mathbf{r}, \mathbf{A r}=$ furan-2-yl, $\mathbf{R}_{1}=\mathbf{C H}_{3}, \mathbf{R}_{2}=$ isopentylamino White powder; Yield $48 \%$; mp: $88-92^{\circ} \mathrm{C}$; IR $(\mathrm{KBr})(v$, $\left.\mathrm{cm}^{-1}\right): 1748,1686,1634(\mathrm{C}=\mathrm{O}$ stretching $), 3283(\mathrm{~N}-\mathrm{H}$ stretching), $3082(\mathrm{C}-\mathrm{H})$; MS-ESI (m/z): $\left[\mathrm{M}^{+}+1\right] 376.94$; ${ }^{1} \mathrm{H}$ NMR $\left(400 \mathrm{MHz}, \mathrm{CDCl}_{3}\right)$ NMR: $\delta$ 0.89-0.91 (d, $J=$ $\left.8 \mathrm{~Hz}, 6 \mathrm{H}, \mathrm{CH}\left(\mathrm{CH}_{3}\right)_{2}\right), 1.42-1.44\left(\mathrm{~d}, J=8 \mathrm{~Hz}, 3 \mathrm{H}, \mathrm{CH}_{3}\right)$ 1.57-1.64 (m, 2H, CH$), 1.72-1.76\left(\mathrm{~m}, 1 \mathrm{H}, \mathrm{CH}\left(\mathrm{CH}_{3}\right)_{2}\right)$, 3.28-3.30 (m, 2H, N-CH $), 3.76\left(\mathrm{~s}, 3 \mathrm{H}, \mathrm{O}-\mathrm{CH}_{3}\right), 4.53(\mathrm{~m}$, $1 \mathrm{H}, \mathrm{CH}), 6.36(\mathrm{~s}, 1 \mathrm{H}$, pyranone- $H), 6.90-7.490(\mathrm{~m}, 3 \mathrm{H}$, Ar- $H$ ), 9.71 (bs, $1 \mathrm{H}, \mathrm{CON} H$ ), 11.18 (bs, $1 \mathrm{H}, \mathrm{N} H$ ).

\section{$\mathbf{3 s}, \mathbf{A r}=$ furan-2-yl, $\mathbf{R}_{\mathbf{1}}=\mathbf{C H}_{3}, \mathbf{R}_{\mathbf{2}}=$ allylamino}

White powder; Yield $50 \%$; mp: $85-88^{\circ} \mathrm{C}$; IR $(\mathrm{KBr})(v$, $\left.\mathrm{cm}^{-1}\right): 1736,1682,1631(\mathrm{C}=\mathrm{O}$ stretching $), 3283(\mathrm{~N}-\mathrm{H}$ stretching), $3086(\mathrm{C}-\mathrm{H})$; MS-ESI (m/z): $\left[\mathrm{M}^{+}+1\right] 347.12$; ${ }^{1} \mathrm{H}$ NMR (400 MHz, $\mathrm{CDCl}_{3}$ ) NMR: $\delta$ 1.42-1.44 (d, $J=$ $\left.8 \mathrm{~Hz}, 3 \mathrm{H}, \mathrm{CH}_{3}\right), 3.70\left(\mathrm{~s}, 1 \mathrm{H}, \mathrm{O}-\mathrm{CH}_{3}\right), 3.93-3.95(\mathrm{~m}, 2 \mathrm{H}$, $\left.\mathrm{CH}_{2}\right), 4.50-4.52(\mathrm{~m}, 1 \mathrm{H}, \mathrm{CH}-\mathrm{N}), 5.19-5.25\left(\mathrm{~m}, 2 \mathrm{H}, \mathrm{CH}_{2}\right.$ (alkene)), 5.79-5.91 (m, 1H, CH), $6.62(\mathrm{~s}, \mathrm{IH}$, pyranone$H)$, 7.03-7.48 (m, 3H, Ar- $H), 9.71$ (bs, $1 \mathrm{H}, \mathrm{CON} H)$, $11.18(\mathrm{bs}, 1 \mathrm{H}, \mathrm{N} H)$.

3t, $\mathbf{A r}=$ furan-2-yl, $\mathbf{R}_{\mathbf{1}}=\mathbf{C H}_{3}, \mathbf{R}_{\mathbf{2}}=$ cycloproylamino White powder; Yield $50 \%$; mp: $119-122{ }^{\circ} \mathrm{C}$; IR $(\mathrm{KBr})(v$, $\left.\mathrm{cm}^{-1}\right): 1747,1685,1581(\mathrm{C}=\mathrm{O}$ stretching $), 3314(\mathrm{~N}-\mathrm{H}$ stretching), $3013(\mathrm{C}-\mathrm{H})$; MS-ESI (m/z): $\left[\mathrm{M}^{+}\right]$346.92; ${ }^{1} \mathrm{H}$ NMR $\left(400 \mathrm{MHz}, \mathrm{CDCl}_{3}\right)$ NMR: $\delta$ 0.66-0.68 $(\mathrm{m}, 2 \mathrm{H}$, $\left.\mathrm{CH}_{2}\right), 0.86-0.88\left(\mathrm{~m}, 2 \mathrm{H}, \mathrm{CH}_{2}\right), 1.42-1.43(\mathrm{~d}, \mathrm{~J}=4 \mathrm{~Hz}, 3 \mathrm{H}$, $\left.\mathrm{CH}_{3}\right), 2.65-2.70(\mathrm{~m}, 1 \mathrm{H}, \mathrm{CH}), 3.70\left(\mathrm{~s}, 3 \mathrm{H}, \mathrm{O}-\mathrm{CH}_{3}\right), 4.47-$ $4.50(\mathrm{~m}, 1 \mathrm{H}, \mathrm{C} H-\mathrm{N}), 6.36(\mathrm{~s}, 1 \mathrm{H}$, pyranone- $H), 7.04-7.50$ $(\mathrm{m}, 3 \mathrm{H}, \operatorname{Ar}-H), 9.71$ (bs, $1 \mathrm{H}, \mathrm{CON} H), 11.18$ (bs, $1 \mathrm{H}$, $\mathrm{N} H)$. 
<smiles>CCOC(=O)c1c(OC)cc(Br)oc1=O</smiles>

$1 \mathrm{f}$<smiles>[R2]c1cc(Br)oc(=O)c1C(=O)OCC</smiles>

3u-x

Scheme S4: Preparation of compounds $3 \mathbf{u}-\mathbf{x}$.

ii) $\mathrm{R}_{2} \mathrm{NH}_{2}$, Ethanol, reflux, 2-6h.

General Procedure: To a solution of appropriate 2a-e $(1.5 \mathrm{mmol})$ in ethanol, appropriate amine $\left(\mathrm{RNH}_{2}, 2.2 \mathrm{mmol}\right)$ was added and refluxed for $2 \mathrm{~h}$ at $80^{\circ} \mathrm{C}$. The completion of the reaction was monitored by TLC. Ethanol was removed under reduced pressure. The crude was dissolved in DCM, washed with water, brine and the organic layer was concentrated under reduced pressure. The crude was purified using column chromatography by eluting with a mixture of hexane and ethylacetate to get $\mathbf{3 u - x}$ in $39-58 \%$ yield.

\section{3u, Ar $=$ 4-F-Ph, $R_{2}=$ isopentylamino}

White powder; Yield 41\%; IR $(\mathrm{KBr})\left(\mathrm{v}, \mathrm{cm}^{-1}\right): 1693$, $1651\left(\mathrm{C}=\mathrm{O}\right.$ stretching), MS-ESI $(\mathrm{m} / \mathrm{z}):\left[\mathrm{M}^{+}\right] 348.10 ;{ }^{1} \mathrm{H}$ NMR $\left(300 \mathrm{MHz}, \mathrm{CDCl}_{3}\right)$ NMR: $\delta, 0.98-1.00(\mathrm{~d}, J=6 \mathrm{~Hz}$, $\left.6 \mathrm{H},\left(\mathrm{CH}_{3}\right)_{2}\right), 1.38-1.43\left(\mathrm{t}, J=6 \mathrm{~Hz}, 3 \mathrm{H}, \mathrm{CH}_{3}\right), 1.58-1.67$ $\left(\mathrm{m}, 4 \mathrm{H},\left(\mathrm{CH}_{2}\right)_{2}\right), 1.72-1.79(\mathrm{~m}, 1 \mathrm{H}, \mathrm{CH}), 3.37-3.44(\mathrm{~m}$, $\left.2 \mathrm{H}, \mathrm{CH}_{2}\right), 4.32-4.39$ (q, $\left.J=6 \mathrm{~Hz}, 2 \mathrm{H}, \mathrm{CH}_{2}\right), 6.36(\mathrm{~s}, 1 \mathrm{H}$, pyranone- $H$ ), 7.13-7.19 (m, 2H, Ar- $H), 7.85-7.89(\mathrm{~m}, 2 \mathrm{H}$, Ar- $H), 10.05(\mathrm{bs}, 1 \mathrm{H}, \mathrm{N} H)$

\section{$3 \mathrm{w}, \mathrm{Ar}=4-\mathrm{F}-\mathrm{Ph}, \mathrm{R}_{\mathbf{2}}=$ cyclohexylamino}

White powder; Yield 39\%; MS-ESI $(\mathrm{m} / \mathrm{z}):\left[\mathrm{M}^{+}+1\right]$ 360.16; ${ }^{1} \mathrm{H}$ NMR $\left(300 \mathrm{MHz}, \mathrm{CDCl}_{3}\right)$ NMR: $\delta$ 1.38-1.43 $(\mathrm{t}, J=6 \mathrm{~Hz}, 3 \mathrm{H}, \mathrm{CH}), 1.57\left(\mathrm{~m}, 2 \mathrm{H}, \mathrm{CH}_{2}\right), 1.46(\mathrm{~m}, 2 \mathrm{H}$, $\left.\mathrm{CH}_{2}\right), 1.64\left(\mathrm{~m}, 2 \mathrm{H}, \mathrm{CH}_{2}\right), 1.83\left(\mathrm{~m}, 2 \mathrm{H}, \mathrm{CH}_{2}\right), 1.99(\mathrm{~m}$, $\left.2 \mathrm{H}, \mathrm{CH}_{2}\right), 3.57(\mathrm{~m}, 1 \mathrm{H}, \mathrm{CH}), 4.32-4.39(\mathrm{q}, J=6 \mathrm{~Hz}, 2 \mathrm{H}$, $\left.\mathrm{CH}_{2}\right), 6.34$ (s, $1 \mathrm{H}$, pyranone- $H$ ), 7.14-7.19 (m, 2H, Ar- $H$ ), 7.83-7.88 (m, 2H, Ar-H), $10.19(\mathrm{bs}, 1 \mathrm{H}, \mathrm{NH})$ 3v, Ar $=$ 4-F-Ph, $R_{2}=$ allylamino

White powder; Yield 45\%; MS-ESI (m/z): $\left[\mathrm{M}^{+}+1\right]$ 318.11; ${ }^{1} \mathrm{H}$ NMR $\left(300 \mathrm{MHz}, \mathrm{CDCl}_{3}\right.$ ) NMR: $\delta$ 1.38-1.43 $\left(\mathrm{t}, J=6 \mathrm{~Hz}, 3 \mathrm{H}, \mathrm{CH}_{3}\right), 4.04-4.08\left(\mathrm{~m}, 2 \mathrm{H}, \mathrm{CH}_{2}\right), 4.33-4.40$ $\left(\mathrm{q}, J=6 \mathrm{~Hz}, 2 \mathrm{H}, \mathrm{CH}_{2}\right), 5.29-5.35\left(\mathrm{~m}, 2 \mathrm{H}, \mathrm{CH}_{2}\right), \quad 5.90-$ $5.99(\mathrm{~m}, 1 \mathrm{H}, \mathrm{CH}),, 6.33(\mathrm{~s}, 1 \mathrm{H}$, pyranone- $H), 7.12-7.18$ $(\mathrm{m}, 2 \mathrm{H}, \operatorname{Ar}-H), 7.81-7.87$ (m, 2H, Ar-H), 10.21 (bs, $1 \mathrm{H}$, $\mathrm{N} H)$

\section{$3 x, A r=4-F-P h, R_{2}=$ cycloproylamino}

White powder; Yield 58\%;, MS-ESI $(\mathrm{m} / \mathrm{z}):\left[\mathrm{M}^{+}+1\right]$ 318.11; ${ }^{1} \mathrm{H}$ NMR $\left(300 \mathrm{MHz}, \mathrm{CDCl}_{3}\right)$ NMR: $\delta \quad 0.71(\mathrm{~m}$, $\left.2 \mathrm{H}, \mathrm{CH}_{2}\right), 0.95\left(\mathrm{~m}, 2 \mathrm{H}, \mathrm{CH}_{2}\right), 1.22-1.27(\mathrm{t}, J=6 \mathrm{~Hz}, 3 \mathrm{H}$, $\left.\mathrm{CH}_{3}\right), 4.16-4.23\left(\mathrm{q}, J=6 \mathrm{~Hz}, 2 \mathrm{H}, \mathrm{CH}_{2}\right), 5.29-5.35(\mathrm{~m}, 2 \mathrm{H}$, $\left.\mathrm{CH}_{2}\right), 7.12(\mathrm{~s}, 1 \mathrm{H}$, pyranone- $H), 7.39-7.45(\mathrm{~m}, 2 \mathrm{H}, \mathrm{Ar}-H)$, 8.01-8.06 (m, 2H, Ar- $H$ ), 9.73 (bs, $1 \mathrm{H}, \mathrm{N} H)$

\section{X-ray crystal structure data for compound $3 \mathrm{~b}$}

$\mathrm{C}_{18} \mathrm{H}_{28} \mathrm{~N}_{2} \mathrm{O}_{5}, \mathrm{M}=342.34$, monoclinic, space group $=\mathrm{P} 21 / \mathrm{c}, \mathrm{a}=9.3013(6) \AA, \mathrm{b}=18.8147$ (1) $\AA, \mathrm{c}=10.3788$

(7) $\AA, \alpha=90^{\circ}, \beta=108.024\left(4^{\circ}\right), \gamma=90^{\circ}, V=1727.2$ (2) $\AA^{3}, T=296 \mathrm{~K}, \mathrm{Z}=4, \mu=0.097 \mathrm{~mm}^{-1}, \mathrm{~F}(000)=$ $720.0, \mathrm{D}_{\mathrm{c}}=1.316 \mathrm{Mg} \mathrm{m}^{-1}$, crystal size: $0.52 \times 0.33 \times 0.16 \mathrm{~mm}, 4306$ reflections measured, 2726 unique, $\mathrm{R}_{1}=$ 0.0519 for $2726 \mathrm{~F}_{\mathrm{o}}>4 \mathrm{sig}\left(\mathrm{F}_{\mathrm{o}}\right)$ and 0.0884 for all 4306 data and 240 parameters with goodness of fit GooF= 1.376. Unit cell determination and intensity data collection $(\theta \max =28.5)$ was performed with $98.4 \%$ completeness. Structure solutions made by direct methods and refinements by full-matrix least-squares methods on $\mathrm{F}^{2}$. (CCDC No: 150756) 


\section{SUPPORTING INFORMATION}
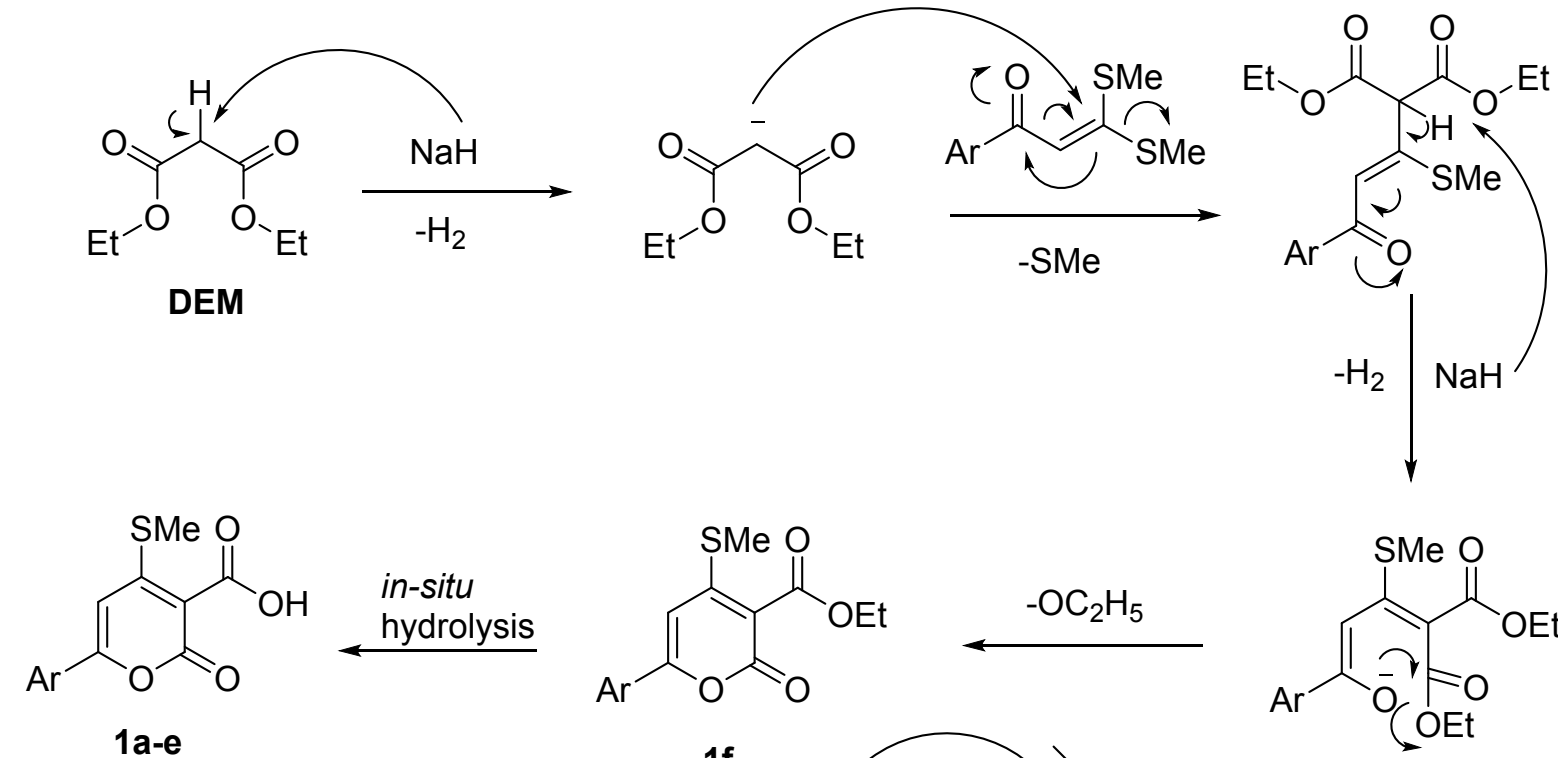<smiles>CSc1cc(Br)oc(=O)c1C(=O)O</smiles>

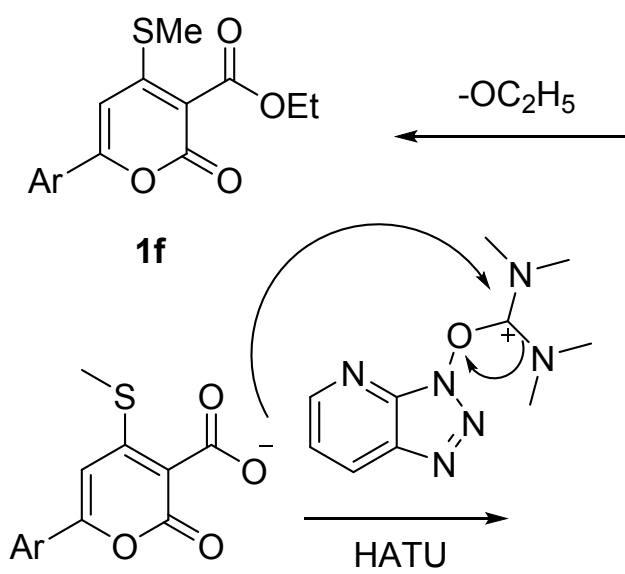<smiles>CCOC(=O)c1cc(Br)cc(S(C)(=O)=O)c1C(=O)OCC</smiles><smiles>[R]NC(=O)c1c(SC)cc([Al])oc1=O</smiles><smiles>[X]C(OC(=O)c1c(SC)cc([Al])oc1=O)C(N(C)C)N(C)C</smiles>

Figure S1: Mechanism of the formation of Pyranone derivatives. 


\section{SUPPORTING INFORMATION}

\section{Analytical spectra of selected compounds}

\section{Compound 1f}

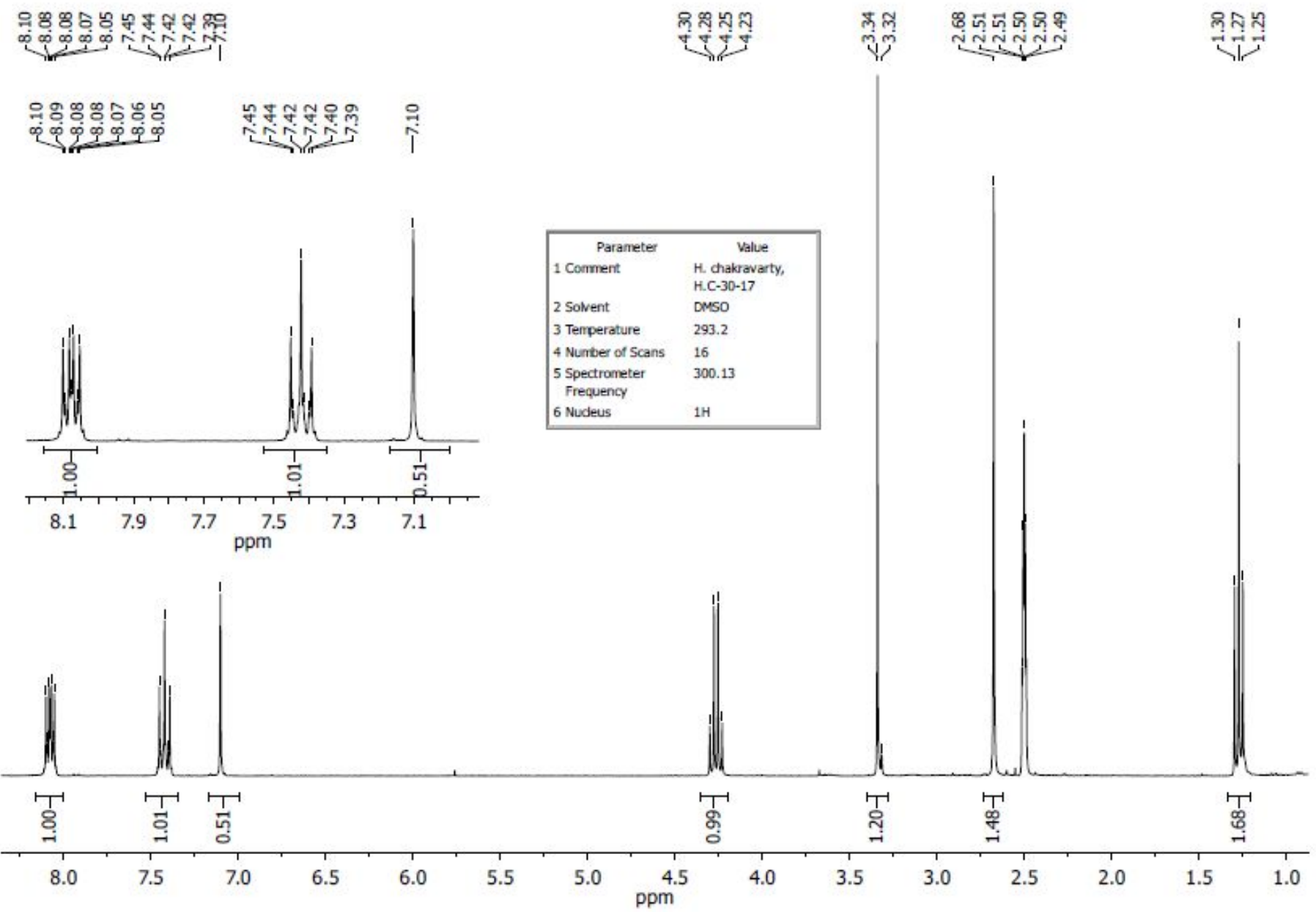

Figure S2: ${ }^{1} \mathrm{H}-\mathrm{NMR}$ spectrum of $\mathbf{1}$ in $\mathrm{DMSO}-\mathrm{d}^{6}$ 


\section{SUPPORTING INFORMATION}

\section{Compound 2a}

SK-III-14-25

File $\mathrm{Na}: 200220329$

Serple Nerno:

Data Collected on:

sople directory

Fidflle; PROTON

Pulse Sequence: PROTON (sepuil)

Solvent: dinso
Data collected on: Mar 292012

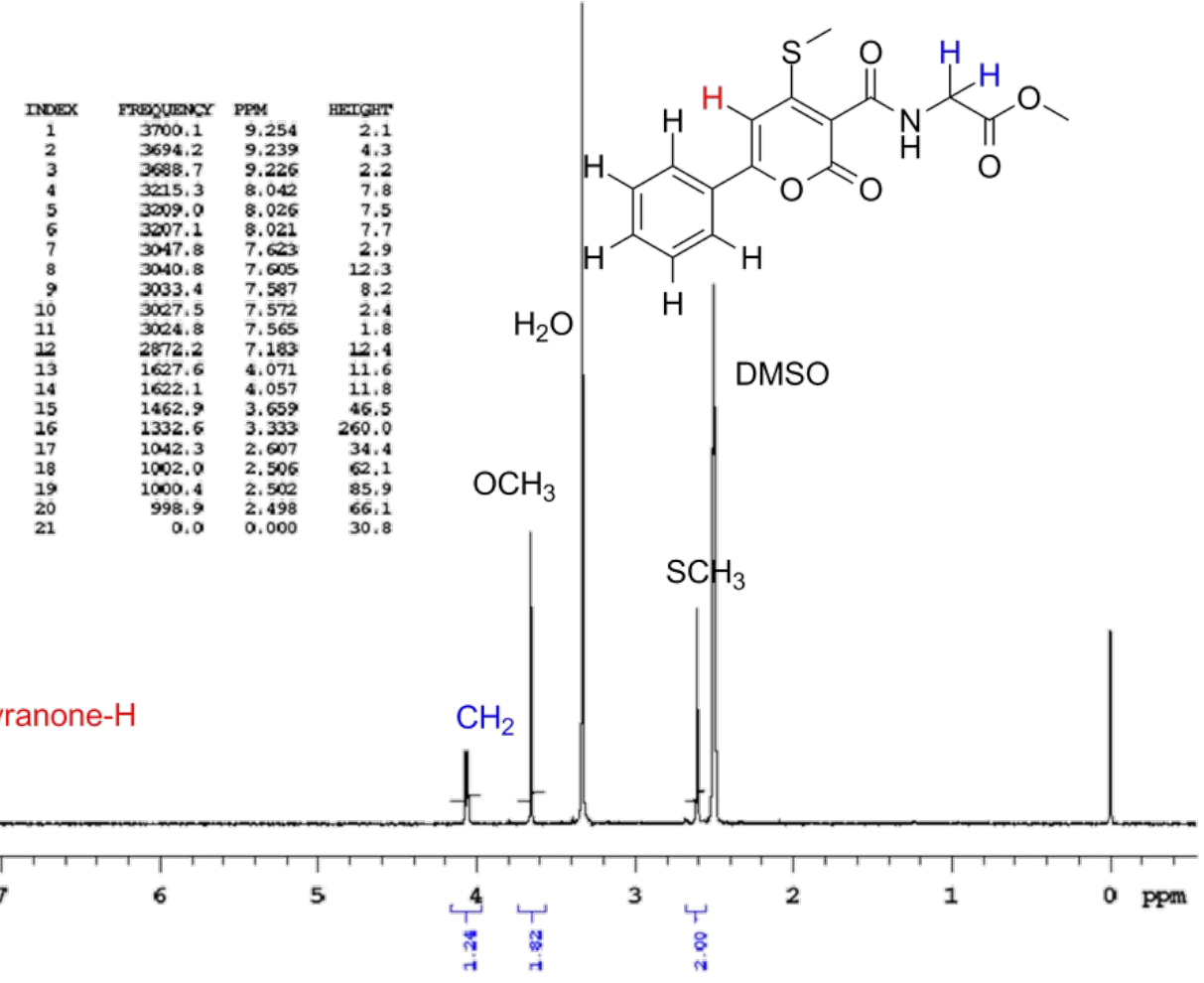

Figure S3: ${ }^{1} \mathrm{H}-\mathrm{NMR}$ spectrum of $\mathbf{2 a}$ in DMSO-d ${ }^{6}$. 


\section{SUPPORTING INFORMATION}

\section{Compound 3p}

Sample Name:

Data Collected on:

Archive directory:

Sample directory:

PIdPIle: PROTON

Pulse Sequence: PROTON ( $82 \mathrm{pul}$ )

Solvent: cdc13
Data collected on: Dec 42013

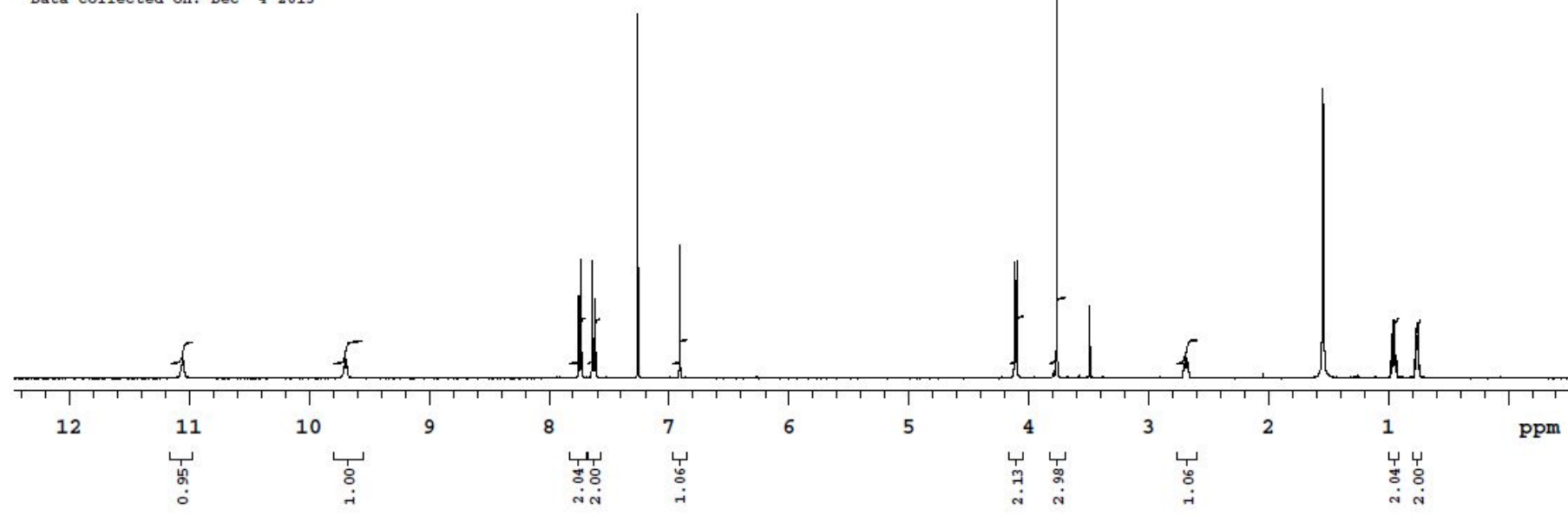

$\begin{array}{crrr}\text { INDEX } & \text { FREQUENCY } & \text { PFM } & \text { HEIGHT } \\ 1 & 4421.6 & 11.059 & 3.3 \\ 2 & 3882.8 & 9.711 & 2.0 \\ 3 & 3877.9 & 9.699 & 3.7 \\ 4 & 3872.2 & 9.685 & 2.1 \\ 5 & 3099.6 & 7.752 & 14.2 \\ 6 & 3090.9 & 7.730 & 20.5 \\ 7 & 3053.2 & 7.636 & 20.1 \\ 8 & 3044.4 & 7.614 & 13.6 \\ 9 & 2902.8 & 7.260 & 62.5 \\ 10 & 2762.5 & 6.909 & 22.9 \\ 11 & 1645.2 & 4.115 & 19.8 \\ 12 & 1639.5 & 4.101 & 20.1 \\ 13 & 1505.8 & 3.766 & 71.6 \\ 14 & 1394.9 & 3.489 & 12.5 \\ 15 & 1079.2 & 2.699 & 2.6 \\ 16 & 1075.2 & 2.689 & 3.5 \\ 17 & 1072.6 & 2.683 & 3.6 \\ 18 & 1069.1 & 2.674 & 2.7 \\ 19 & 617.0 & 1.543 & 49.7 \\ 20 & 391.2 & 0.978 & 2.4 \\ 21 & 384.2 & 0.961 & 9.9 \\ 22 & 378.9 & 0.948 & 10.1 \\ 23 & 371.9 & 0.930 & 3.0 \\ 24 & 310.5 & 0.777 & 3.1 \\ 25 & 305.3 & 0.764 & 8.5 \\ 26 & 303.1 & 0.758 & 9.4 \\ 27 & 301.8 & 0.755 & 8.5 \\ 28 & 300.0 & 0.750 & 7.7 \\ 29 & 294.3 & 0.736 & 2.2\end{array}$

Figure S4: ${ }^{1} \mathrm{H}-\mathrm{NMR}$ spectrum of $\mathbf{3 p}$ in $\mathrm{CDCl}_{3}$. 


\section{SUPPORTING INFORMATION}

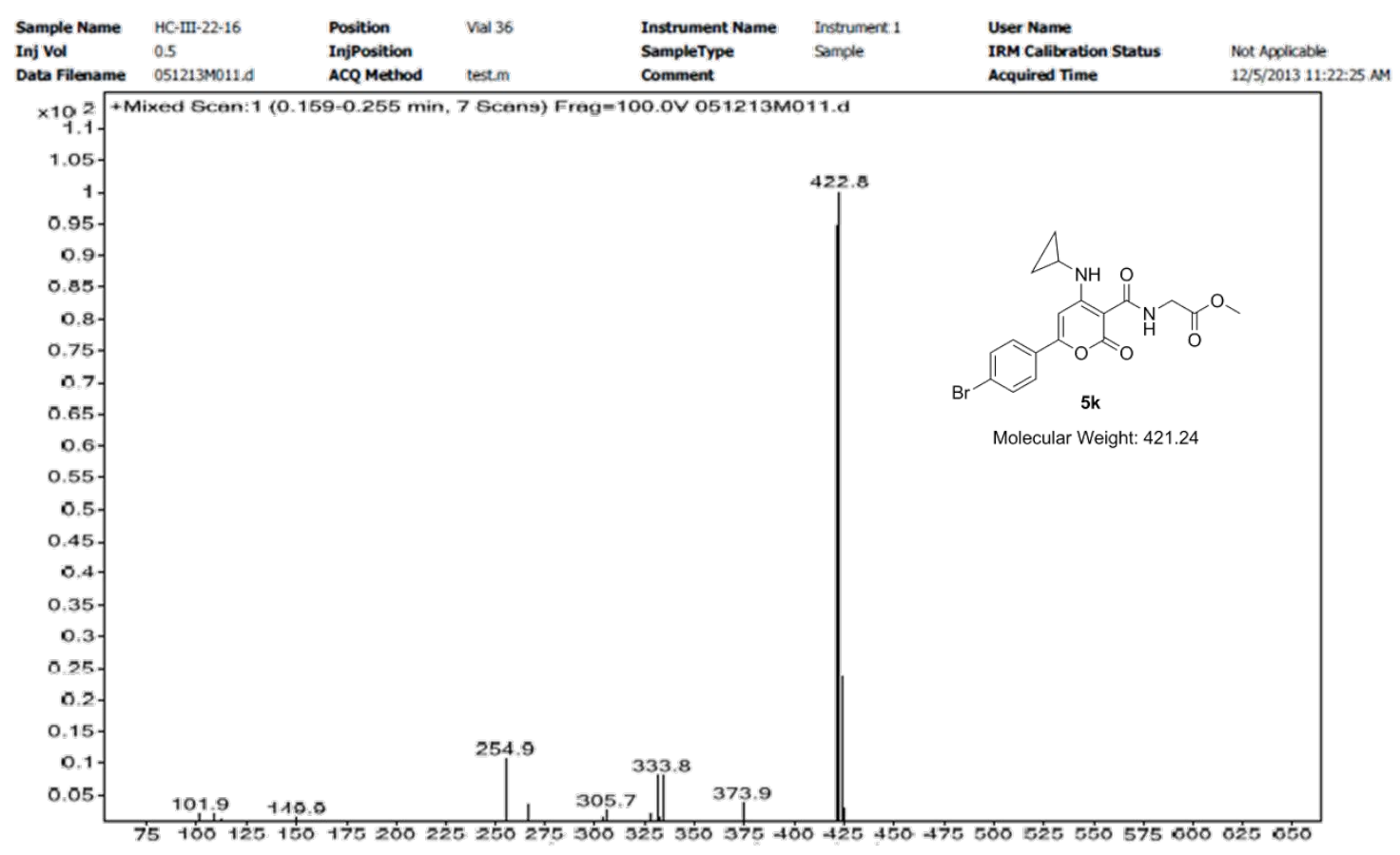

Figure S5: Mass spectrum of $\mathbf{3 p}$.

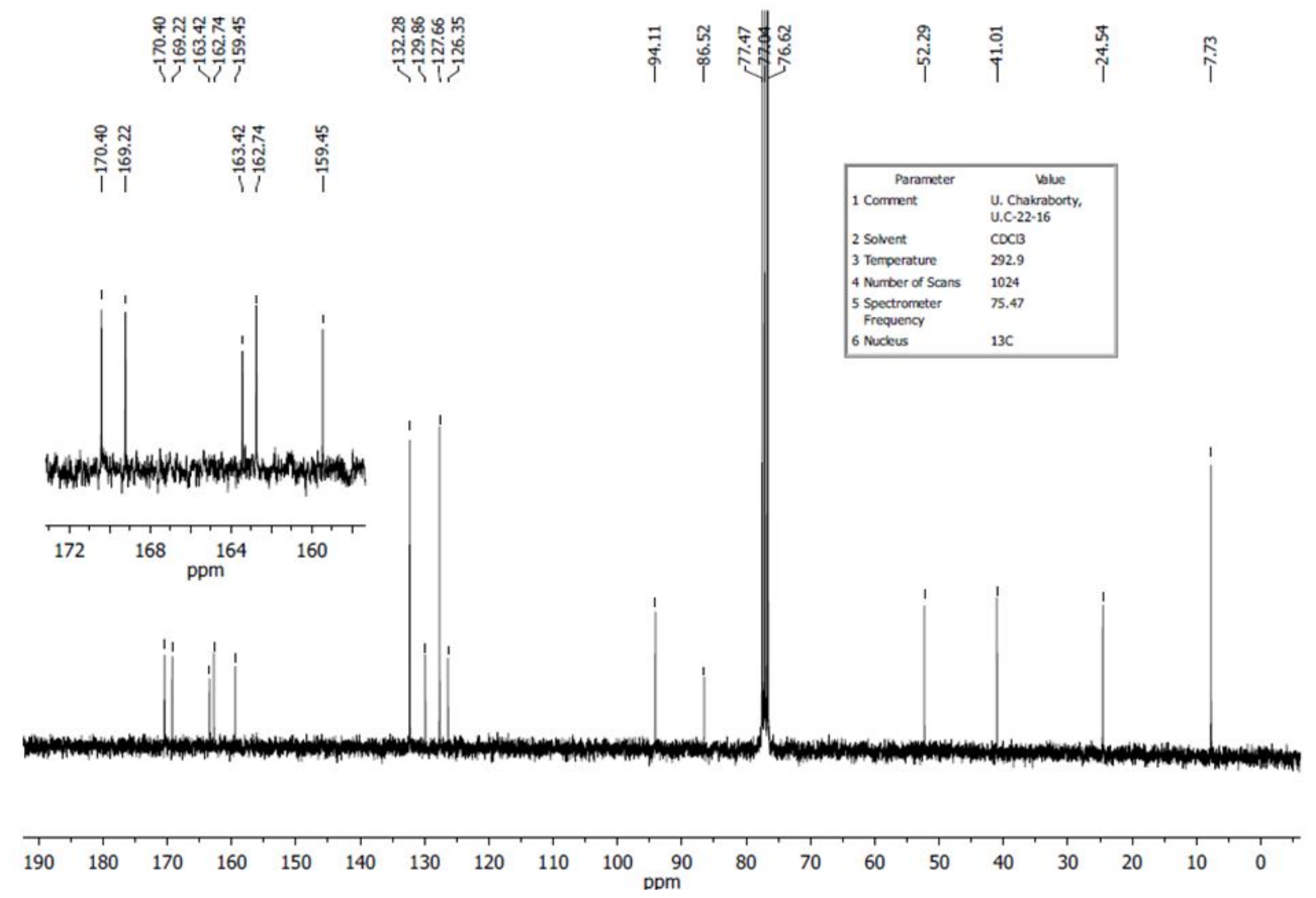

Figure S6: ${ }^{13} \mathrm{C}-\mathrm{NMR}$ spectrum of $\mathbf{3 p}$ in $\mathrm{CDCl}_{3}$. 


\section{SUPPORTING INFORMATION}

\section{Compound 3d}

\begin{tabular}{|ll|}
\hline \multicolumn{1}{|c|}{ Parameter } & \multicolumn{1}{c|}{ Velue } \\
1 Comment & H. chalsavarty, H.C-37-18 \\
2 Solvent & CDCB \\
3 Temperature & 293.3 \\
4 Number of SCans & 16 \\
5 Spectrometer Frequency 300.13 \\
6 Nucleus & $1 H$ \\
\hline
\end{tabular}
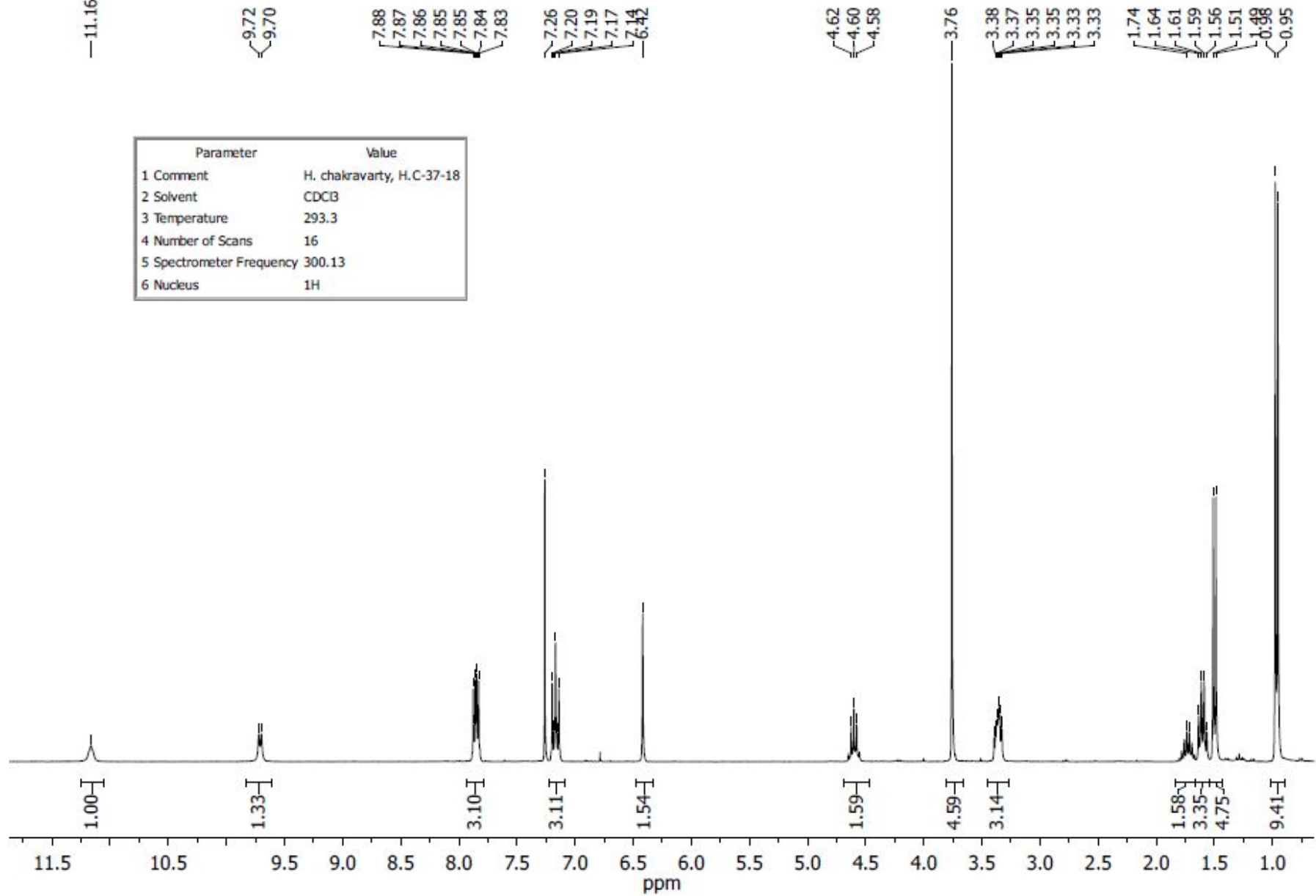

Figure S7: ${ }^{1} \mathrm{H}-\mathrm{NMR}$ spectrum of $\mathbf{3 d}$ in $\mathrm{CDCl}_{3}$. 


\section{SUPPORTING INFORMATION}

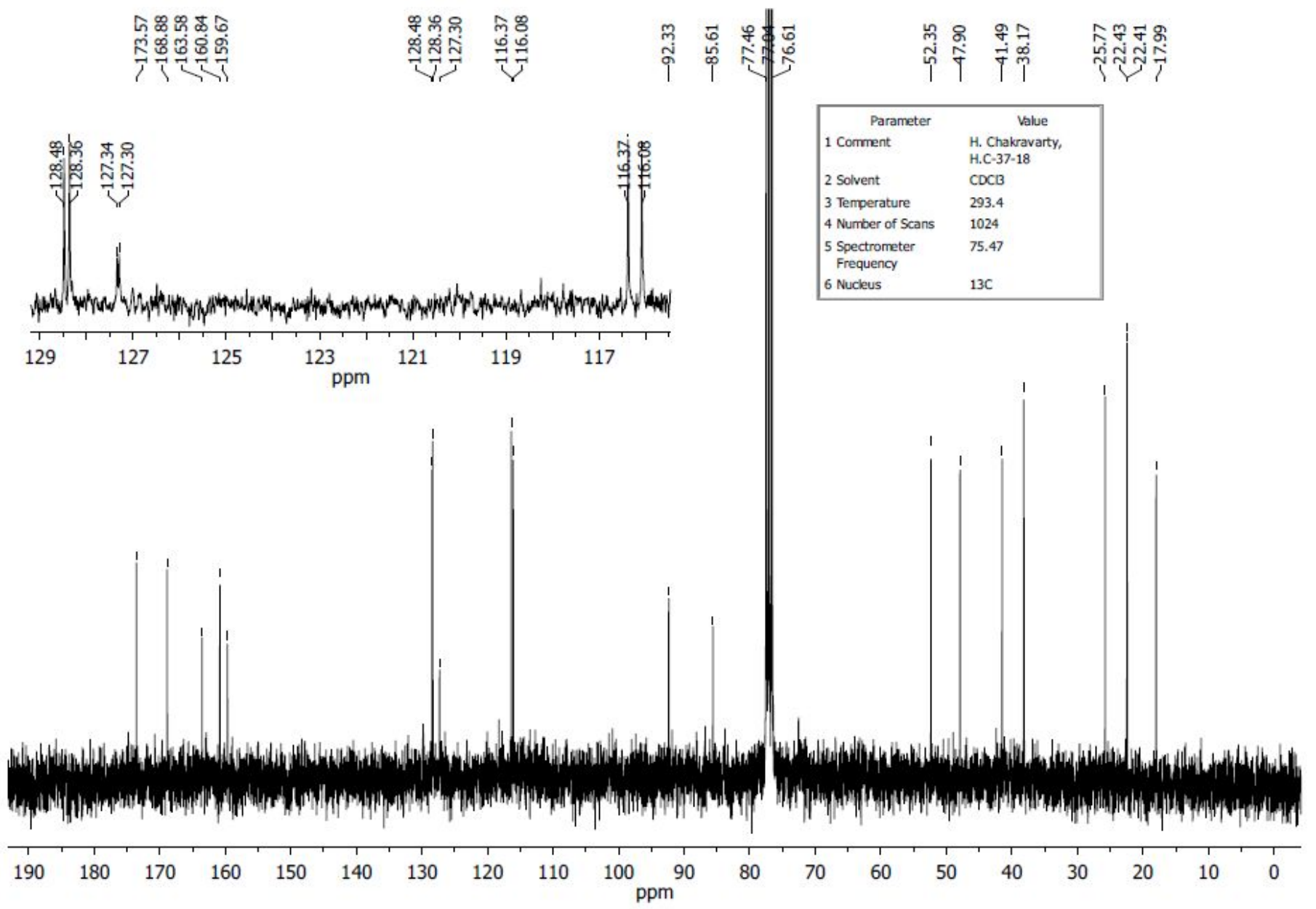

Figure S8: ${ }^{13} \mathrm{C}-\mathrm{NMR}$ spectrum of $\mathbf{3 d}$ in $\mathrm{CDCl}_{3}$. 


\section{SUPPORTING INFORMATION}

Compound 30
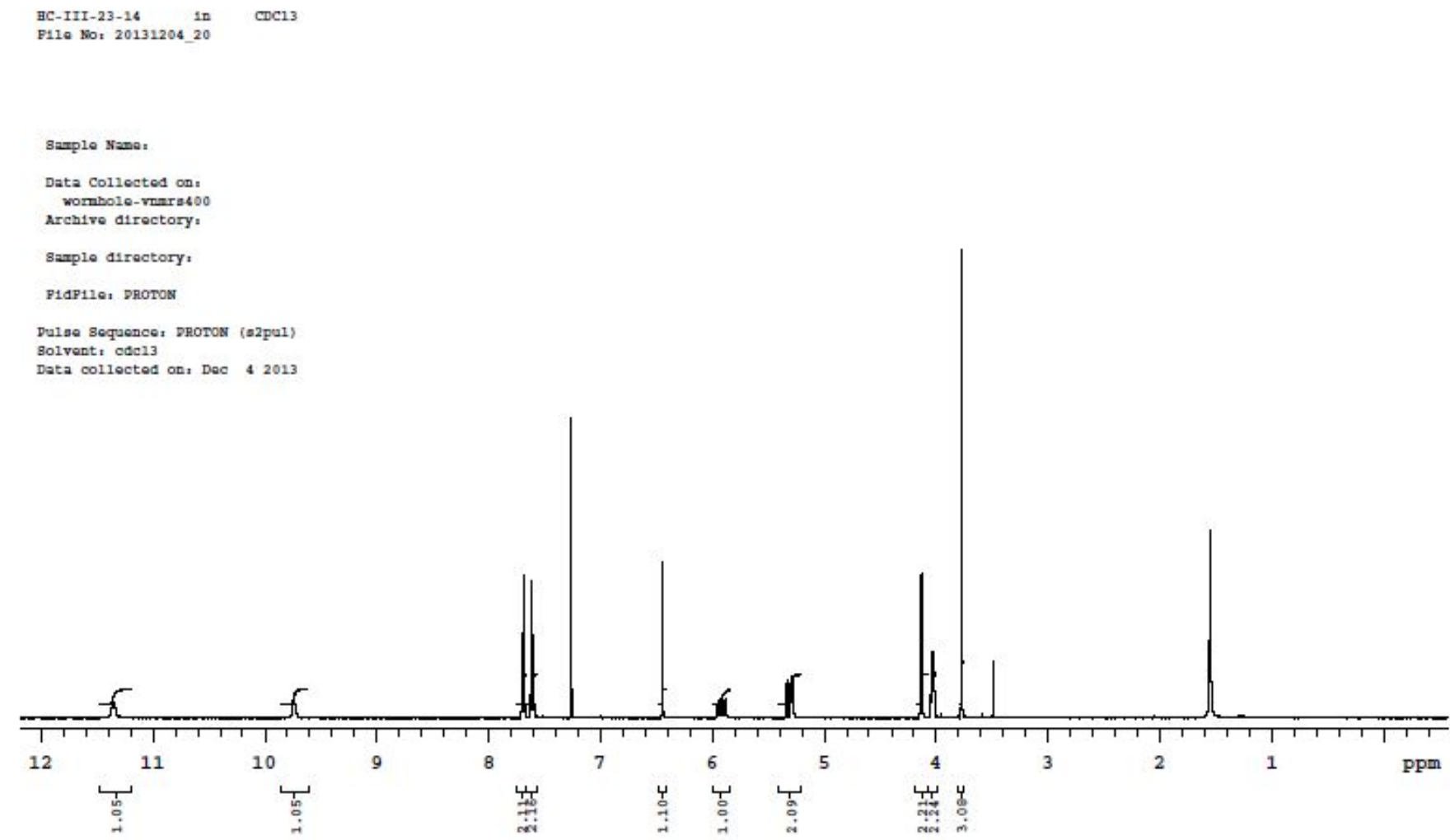

Figure S9: ${ }^{1} \mathrm{H}-\mathrm{NMR}$ spectrum of $3 \mathrm{o}$ in $\mathrm{CDCl}_{3}$. 


\section{SUPPORTING INFORMATION}

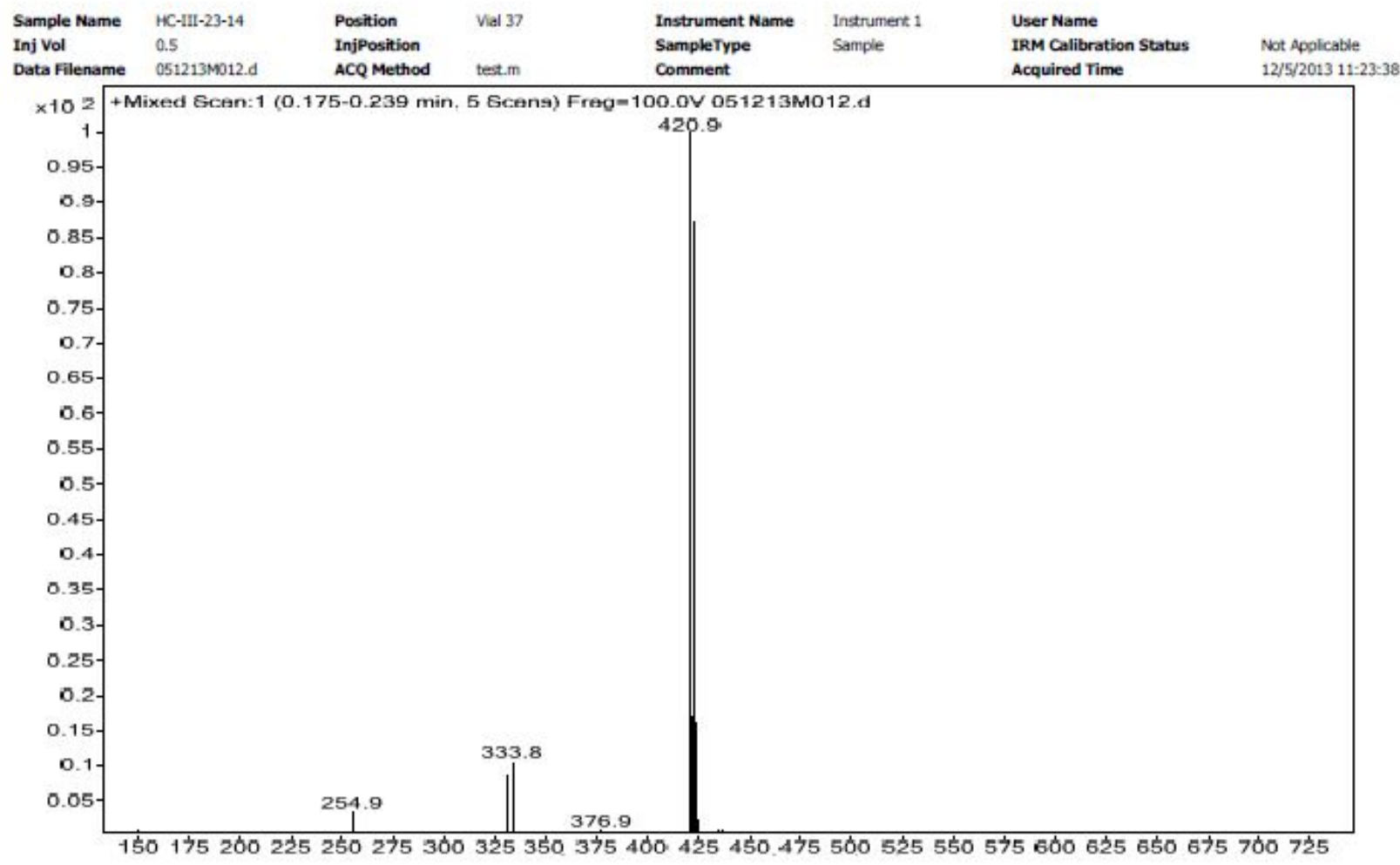

Figure S10: Mass spectrum of $\mathbf{3 0}$. 


\section{SUPPORTING INFORMATION}

\section{Compound 3m}

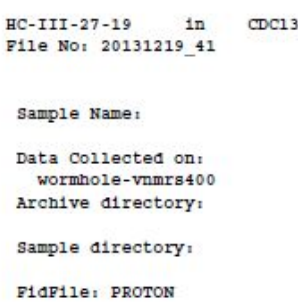

Pulse Sequence: PROTON (s2pul) Solvent: cac13

Data collected on: Dec 192013

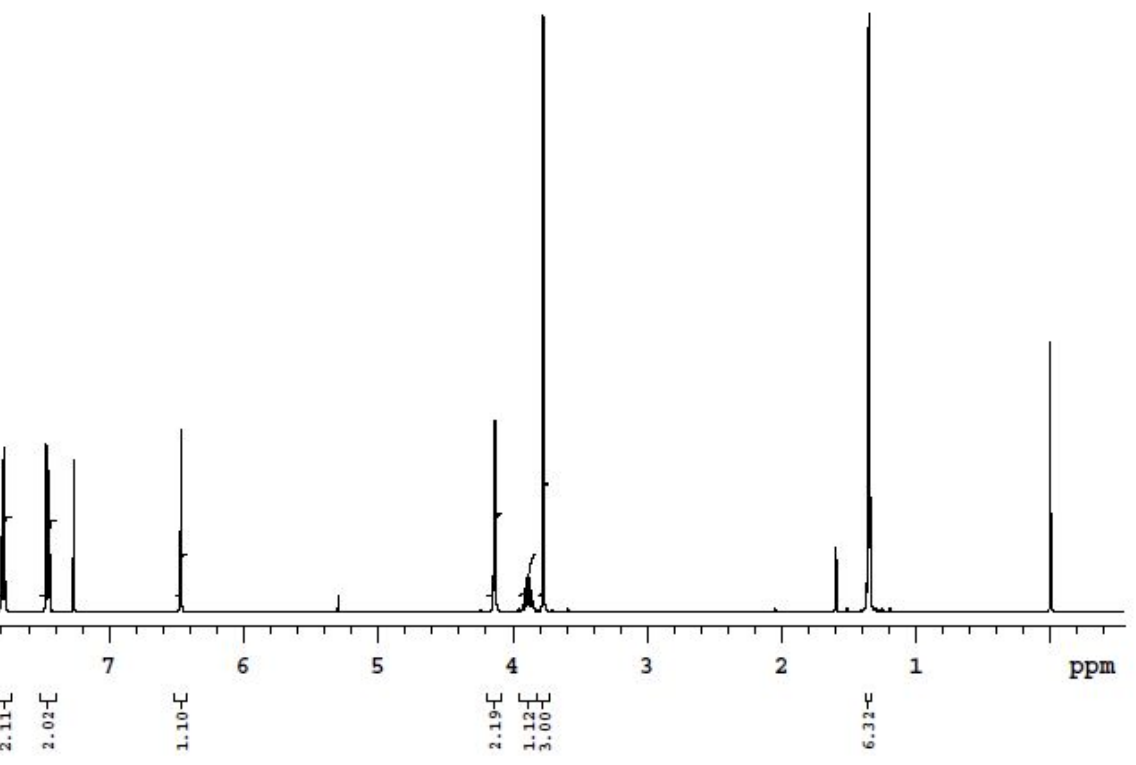

\begin{tabular}{crrr} 
INDEX & PREQUENCY & \multicolumn{1}{l}{ PPM } & HEIGHT \\
1 & 4490.4 & 11.231 & 3.4 \\
2 & 4484.7 & 11.216 & 3.4 \\
3 & 3912.5 & 9.785 & 3.3 \\
4 & 3907.2 & 9.772 & 6.0 \\
5 & 3902.0 & 9.759 & 3.5 \\
6 & 3117.1 & 7.796 & 23.5 \\
7 & 3108.3 & 7.774 & 25.5 \\
8 & 2984.7 & 7.465 & 26.0 \\
9 & 2975.9 & 7.443 & 22.1 \\
10 & 2904.0 & 7.263 & 23.4 \\
11 & 2584.8 & 6.465 & 28.4 \\
12 & 1655.7 & 4.141 & 29.5 \\
13 & 1650.0 & 4.127 & 29.7 \\
14 & 1561.8 & 3.906 & 3.4 \\
15 & 1555.3 & 3.890 & 5.3 \\
16 & 1548.2 & 3.872 & 5.3 \\
17 & 1541.7 & 3.856 & 3.5 \\
18 & 1507.9 & 3.771 & 92.4 \\
19 & 636.7 & 1.592 & 10.1 \\
20 & 543.7 & 1.360 & 90.6 \\
21 & 537.1 & 1.343 & 92.7 \\
22 & -0.4 & -0.001 & 41.7
\end{tabular}

Figure S11: ${ }^{1} \mathrm{H}-\mathrm{NMR}$ spectrum of $\mathbf{3 m}$ in $\mathrm{CDCl}_{3}$. 


\section{SUPPORTING INFORMATION}

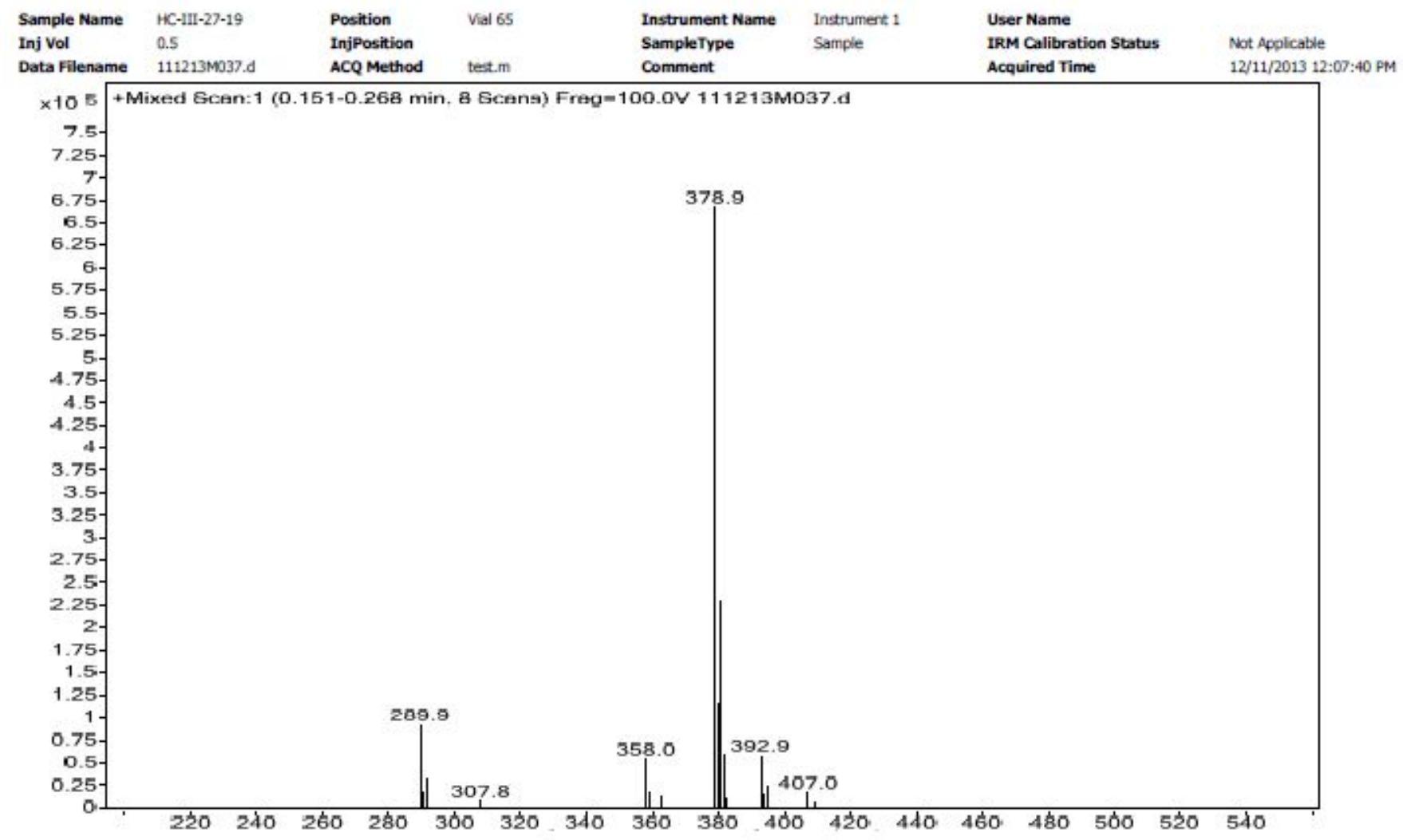

Figure S12: Mass spectrum of $\mathbf{3 m}$. 


\section{SUPPORTING INFORMATION}

\section{Compound 3u}

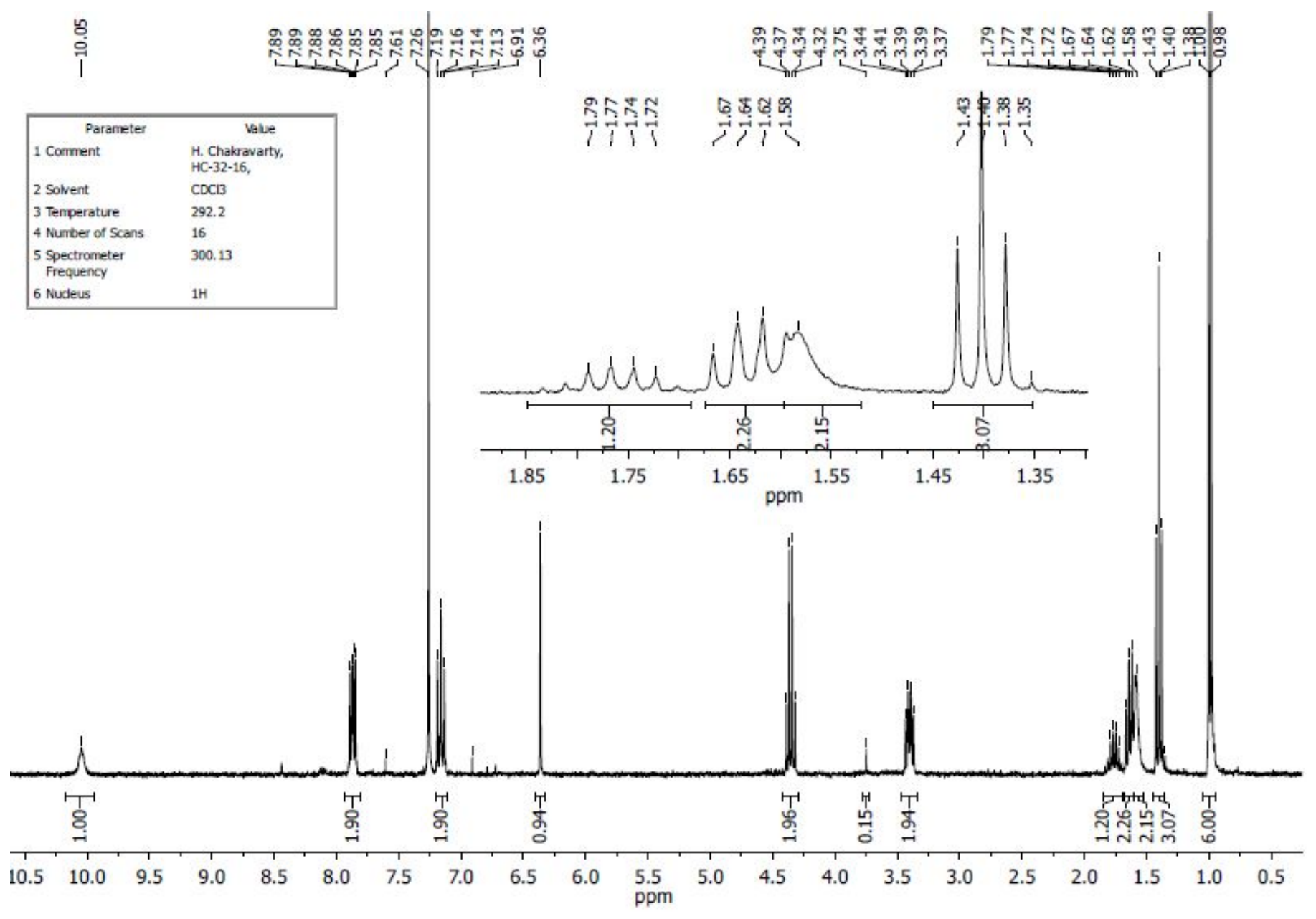

Figure S13: ${ }^{1} \mathrm{H}-\mathrm{NMR}$ spectrum of $\mathbf{3 u}$ in $\mathrm{CDCl}_{3}$. 


\section{SUPPORTING INFORMATION}

\section{Compound 3v}

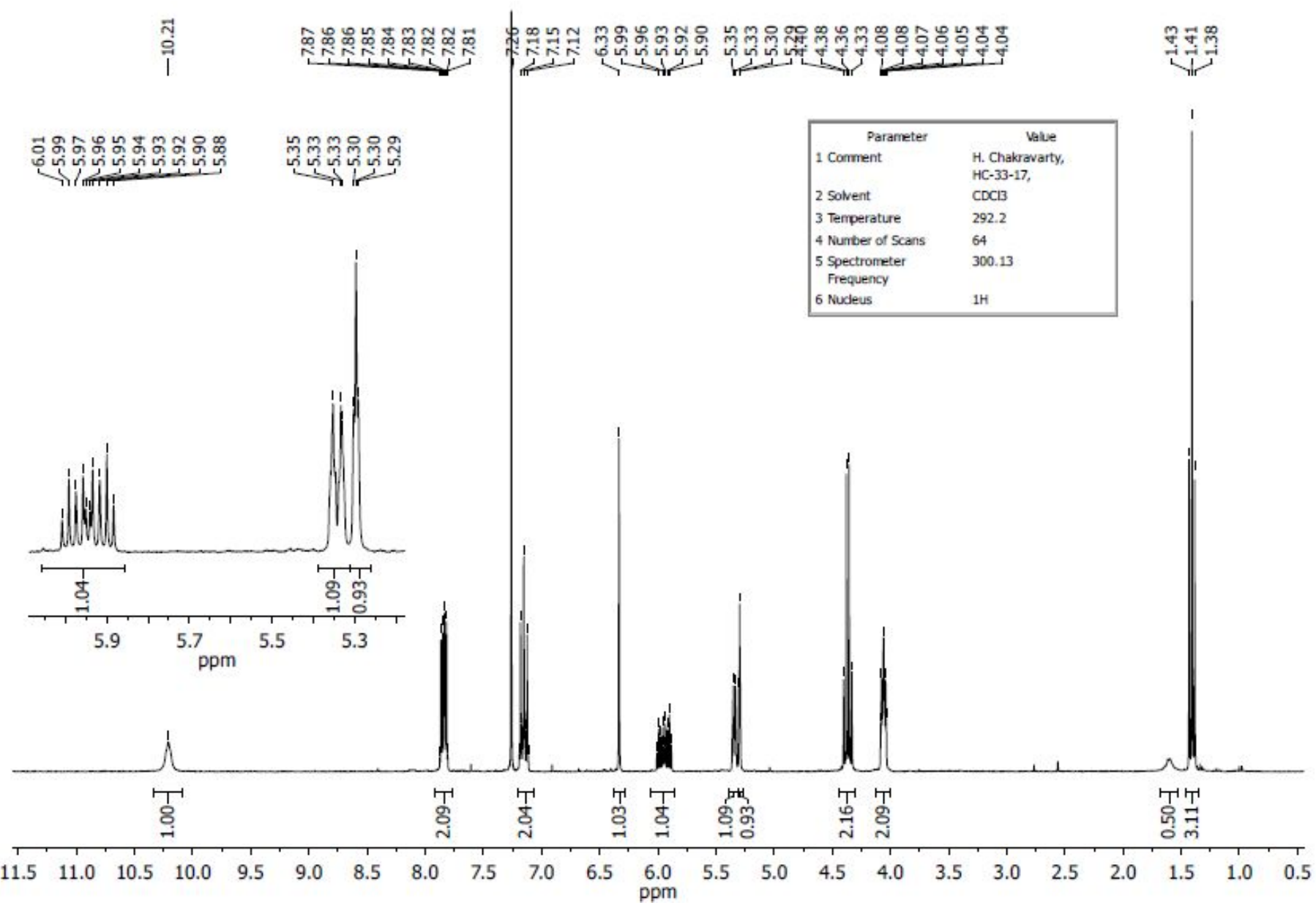

Figure S14: ${ }^{1} \mathrm{H}-\mathrm{NMR}$ spectrum of $\mathbf{3 v}$ in $\mathrm{CDCl}_{3}$. 